Pacific

Journal of

Mathematics

RESIDUAL SPECTRUM OF SPLIT CLASSICAL GROUPS; CONTRIBUTION FROM BOREL SUBGROUPS

HENRY H. KIM

Volume 199 No. 2

June 2001 


\title{
RESIDUAL SPECTRUM OF SPLIT CLASSICAL GROUPS; CONTRIBUTION FROM BOREL SUBGROUPS
}

\author{
HENRY H. KIM
}

\begin{abstract}
We completely determine the residual automorphic representations coming from the torus of odd orthogonal groups. Under certain technical assumption on disconnected groups, our results are valid for symplectic groups and even orthogonal groups. Moeglin has determined the residual spectrum attached to the trivial character of the torus for split classical groups. However, non-trivial characters present some nontrivial difficulties, both local and global. Local problems are resolved in a companion paper with C. Jantzen, where many other useful results are obtained.
\end{abstract}

\section{Introduction.}

Let $F$ be a number field and $\mathbb{A}$ its ring of adeles. Let $G$ be a reductive group defined over $F$. For simplicity we assume that the center of $G$ is anisotropic over $F$. A central problem in the theory of automorphic forms is to decompose the right regular representation of $G(\mathbb{A})$ acting on the Hilbert space $L^{2}(G(F) \backslash G(\mathbb{A}))$. It has continuous spectrum and discrete spectrum.

$$
L^{2}(G(F) \backslash G(\mathbb{A}))=L_{\text {dis }}^{2}(G(F) \backslash G(\mathbb{A})) \oplus L_{\text {cont }}^{2}(G(F) \backslash G(\mathbb{A})) .
$$

We are mainly interested in the discrete spectrum. Main contributions have been made by Langlands and Arthur. First of all Langlands described, using his theory of Eisenstein series, an orthogonal decomposition of this space of the form:

$$
L_{\text {dis }}^{2}(G(F) \backslash G(\mathbb{A}))=\bigoplus_{(M, \pi)} L_{\text {dis }}^{2}(G(F) \backslash G(\mathbb{A}))_{(M, \pi)},
$$

where $(M, \pi)$ is a Levi subgroup with a cuspidal automorphic representation $\pi$ taken modulo conjugacy (here we normalize $\pi$ so that the action of the maximal split torus in the center of $G$ at the archimedean places is trivial) and $L_{\text {dis }}^{2}(G(F) \backslash G(\mathbb{A}))_{(M, \pi)}$ is a space of residues of Eisenstein series associated to $(M, \pi)$. Here we note that the subspace

$$
\oplus_{(G, \pi)} L_{\text {dis }}^{2}(G(F) \backslash G(\mathbb{A}))_{(G, \pi)},
$$

is the space of cuspidal representations $L_{\text {cusp }}^{2}(G(F) \backslash G(\mathbb{A}))$. Its orthogonal complement in $L_{\text {dis }}^{2}(G(F) \backslash G(\mathbb{A}))$ is called the residual spectrum and we 
denote it by $L_{\text {res }}^{2}(G(F) \backslash G(\mathbb{A}))$. Therefore we have an orthogonal decomposition

$$
L_{\text {dis }}^{2}(G(F) \backslash G(\mathbb{A}))=L_{\text {cusp }}^{2}(G(F) \backslash G(\mathbb{A})) \oplus L_{\text {res }}^{2}(G(F) \backslash G(\mathbb{A})) .
$$

Arthur described, motivated by his trace formula, described a conjectural decomposition of this space as follows:

$$
L_{\text {dis }}^{2}(G(F) \backslash G(\mathbb{A}))=\oplus_{\psi} L^{2}(G(F) \backslash G(\mathbb{A}))_{\psi},
$$

where $\psi$ runs, modulo conjugacy, through the set of morphism $\psi: L_{F} \times$ $S L_{2}(\mathbb{C}) \longmapsto G^{*}$, where $L_{F}$ is the conjectural tannakian group, $G^{*}$ is the Langlands' $L$-group and $\psi$ satisfies certain conditions; in particular, $\psi$ restricted to $S L_{2}(\mathbb{C})$ is algebraic, $\psi$ restricted to $L_{F}$ parametrizes a cuspidal tempered representation of a Levi subgroup and the image of $\psi$ is not included in proper Levi subgroups. The space $L^{2}(G(F) \backslash G(\mathbb{A}))_{\psi}$ is defined by local data (3.3).

Let $\Pi_{\text {res } v}$ be the set of the local components of the residual spectrum $L_{\text {dis }}^{2}(G(F) \backslash G(\mathbb{A}))_{(M, \pi)}$. It is of great importance to prove that $\Pi_{\text {res } v} \subset$ $\Pi_{\psi_{v}}$ for some $\psi_{v}$ and the multiplicity formula (3.1) holds: In other words, the Arthur parameters parametrize the residual spectrum. For this, it is necessary to construct a set of characters $C_{\text {res }_{v}} \subset \widehat{C_{\psi_{v}}}$ attached to $\Pi_{\text {res }_{v}}$.

We should note that Arthur's trace formula will not separate the cuspidal part from the residual one and therefore one needs to study the residual spectrum by computing the residues of Eisenstein series. It is noted that the first exotic example of the residual spectrum of the split group $G_{2}$, which was discovered by Langlands and further studied later by Moeglin and Waldspurger, has been of significance to Arthur in formulating his conjectures.

There are two problems in calculating the residual spectrum. The first problem is global. It is concerned with calculating the residues of the Eisenstein series via the constant term of the Eisenstein series. Computing residues of Eisenstein series is a very difficult problem and requires both the knowledge of the poles of corresponding automorphic $L$-functions, as well as handling very difficult computational combinatorics. These computations are already highly non-trivial even for rank 2 split groups. For example, for the split group $G_{2}$, the Eisenstein series, built out of the maximal parabolic subgroup attached to the long simple root, contains the third symmetric power $L$-function of $G L_{2}$. The precise location of the poles of the third symmetric power $L$-function was resolved only in the recent work of [Ki-Sh2] (see the introduction of the paper for the long history). The Eisenstein series built out of the minimal parabolic subgroups do not present a problem with regards to poles of automorphic $L$-functions since the $L$-functions are Hecke $L$-functions. However, in this case, cancelation of poles of the normalized intertwining operators presents a problem. Even for $S p_{4}$ [Ki1], one has to use a non-trivial fact on the subtle analysis of the normalized intertwining 
operators for $S L_{2}$ due to Labesse-Langlands [L-La]. The second problem is local. It is concerned with calculating the image of the normalized local intertwining operators of the generalized principal series. While the unitary principal series are well understood thanks to the Knapp-Stein $R$-group theory, the generalized principal series are not. Moeglin's result [M1] on the unramified principal series of the split classical groups seems to be most satisfactory so far.

At this moment, the most satisfying result about the residual spectrum is due to Moeglin-Waldspurger [M-W2] who completely determined the residual spectrum for $G L_{n}$ and Arthur [A2] gave the Arthur parameters for them. Their result is that the residual spectrum for $G L_{n}$ is parametrized by the cuspidal representations of $G L_{m}$ and the principal unipotent orbit of $G L_{\frac{n}{m}}(\mathbb{C})$, where $m \mid n$. As we observed, Arthur's parameters require the introduction of the hypothetical group $L_{F}$. However, there are parts of it that can be parametrized by our existing knowledge. This is the case in particular, if the Eisenstein series is built out of minimal parabolics. In this case, $L_{F}$ can be replaced by the global Weil group $W_{F}$. Therefore, we will restrict ourselves to the study of $L_{\text {dis }}^{2}(G(F) \backslash G(\mathbb{A}))_{(T, \chi)}$, where $T$ is a maximal split torus and $\chi$ is a unitary character of $T$.

When $G$ is a split classical group and $\chi$ is trivial, Moeglin [M1] completely solved the problem with the restriction that archimedean components are spherical. In this case the Arthur parameter is given by

$$
\psi: W_{F} \times S L_{2}(\mathbb{C}) \longmapsto G^{*}
$$

where $\left.\psi\right|_{W_{F}}=1$ and $\left.\psi\right|_{S L_{2}(\mathbb{C})}$ is determined by a distinguished unipotent orbit of $G^{*}$. Recall that the Springer correspondence is an injective map from the set of irreducible characters of $W$, the Weyl group, into the set of pairs $(O, \eta)$, where $O$ is a unipotent orbit and $\eta$ is an irreducible character of $A(u)=C(u) / C(u)^{0}, u \in O$, where $C(u)$ is the centralizer of $u$ in $G^{*}$. Let Springer $(O)$ be the set of characters of $A(u)$ which are in the image of the Springer correspondence. Then Moeglin's result is that the local components of the residual spectrum are parametrized by the distinguished unipotent orbits $O$ of $G^{*}$ and Springer $(O)$. Let $\operatorname{Unip}(O)$ be the set of the local components of the residual spectrum. Then there is a pairing between $\operatorname{Unip}(O)$ and Springer $(O)$ which gives a desired Arthur's multiplicity formula (3.2).

Moeglin [M4] also constructed the local representations associated to the remaining characters of $A(u)$ which are NOT in Springer $(O)$. These should be local components of cuspidal representations. She [M5] obtained a partial result on how to determine when $\pi \in \Pi_{\psi}$ is a cuspidal representation. It would be an important but a difficult problem. We note that Lusztig [Lu] 
has a theory of the generalized Springer correspondence which gives the remaining characters of $A(u)$.

In this paper, we will extend Moeglin's result when $\chi$ is an arbitrary unitary character of $T$. For simplicity, we will describe the result for $G=$ $S p_{2 n}$. Because the result in [Ja-Ki] for $S p_{2 n}, O_{2 n}$ is not complete, our result for $S p_{2 n}$ is not complete. This is due to the fact that Barbasch-Moy's result [B-Mo2] is not available for disconnected groups like $O_{2 n}$. However, we obtain a complete result for $S_{2 n+1}$. Nevertheless, the symplectic group case will illustrate the technique and the heart of the matter.

As in $[\mathbf{M} 1]$, we use $G^{*}=O_{2 n+1}(\mathbb{C})$ to denote its dual group. Let $\mu_{1}, \ldots, \mu_{k}$ be $k$ distinct non-trivial quadratic grössencharacters of $F$. Fix integers $r_{1} \geq \cdots \geq r_{k} \geq 2$ and $r_{0}$ so that $r_{0}+r_{1}+\cdots+r_{k}=n$. Then $\chi=\chi(\underbrace{\mu_{1}, \ldots, \mu_{1}}_{r_{1}}, \ldots, \underbrace{\mu_{k}, \ldots, \mu_{k}}_{r_{k}}, \underbrace{1, \ldots, 1}_{r_{0}})$. We showed in $[\mathbf{K i}-\mathbf{S h}]$ that only the character of the above type contributes to the residual spectrum. The character $\chi$ defines a homomorphism of the Weil group $W_{F}$ into a Cartan subgroup of $S O_{2 n+1}(\mathbb{C})$. Composing this homomorphism with the standard action of $O_{2 n+1}(\mathbb{C})$ on $\mathbb{C}^{2 n+1}$ will then give a completely reducible representation of $W_{F}$ on $\mathbb{C}^{2 n+1}$ which decomposes according to eigenvalues $\mu_{1}, \ldots, \mu_{k}$, and 1 , with multiplicities $2 r_{1}, \ldots, 2 r_{k}$, and $2 r_{0}+1$, respectively. Write $\mathbb{C}^{2 n+1}=V_{0} \oplus V_{1} \oplus \ldots \oplus V_{k}$, where each $V_{i}$, $\operatorname{dim} V_{i}=2 r_{i}$, is the eigenspace attached to eigenvalue $\mu_{i}, 1 \leq i \leq k$, and $V_{0}$ is the trivial eigenspace of dimension $2 r_{0}+1$. In this way we get an embedding of $\prod_{i=0}^{k} O\left(V_{i}\right) \subset O_{2 n+1}(\mathbb{C})$. For each $i, 0 \leq i \leq k$, let $O_{i}$ be the distinguished unipotent orbit of $O\left(V_{i}\right)$. Then Arthur parameter of interest to us is a homomorphism

$$
\psi: W_{F} \times S L_{2}(\mathbb{C}) \longmapsto \prod_{i=0}^{k} O\left(V_{i}\right) \subset O_{2 n+1}(\mathbb{C}),
$$

satisfying,

(1) $\psi \mid W_{F}: w \longmapsto 1 \times \mu_{1}(w) \times \cdots \times \mu_{k}(w) \in\{ \pm 1\} \times\{ \pm 1\} \times \cdots \times\{ \pm 1\}$, where $\{ \pm 1\}$ is the center of $O\left(V_{i}\right)$ for $i=0, \ldots, k$.

(2) By Jacobson-Morozov theorem, $\left.\psi\right|_{S L_{2}(\mathbb{C})}$ defines the unipotent orbit $\prod_{i=0}^{k} O_{i}$ of $G^{*}$.

The unipotent orbits $O_{i}$ determine, through the Jacobson-Morozov's theorem, certain conjugacy class of unramified characters of $T$. Let $\lambda_{0}=$ $\lambda_{1,0}+\ldots+\lambda_{k, 0}+\lambda_{0,0}$ be the one in the positive Weyl chamber. To $\psi$, Arthur associates a Langlands' parameter $\phi_{\psi}$ :

$$
\phi_{\psi}: W_{F} \longrightarrow O_{2 n+1}(\mathbb{C}),
$$


where $\phi_{\psi}(w)=\psi\left(w,\left(\begin{array}{cc}|w|^{\frac{1}{2}} & 0 \\ 0 & |w|^{-\frac{1}{2}}\end{array}\right)\right)$. It is given by $\phi_{\psi}=\chi \otimes \exp \left\langle\lambda_{0}\right.$, $\left.H_{B}()\right\rangle$. Its non-tempered part is $\phi_{\psi}^{+}=\exp \left\langle\lambda_{0}, H_{B}()\right\rangle$.

In $[\mathbf{K i}-\mathbf{S h}]$, we studied the special case when $O_{i}$ is the unipotent orbit of the form $\left(2 r_{i}-1,1\right)$ for $i=1, \ldots, k$ and $O_{0}$ is of the form $\left(2 r_{0}+1\right)$. This is the opposite of the trivial character case. In this case, we showed that the local components of the residual spectrum in $\Pi_{\phi_{\psi}}$ are parametrized by the Knapp-Stein $R$-group of the unitary principal series $I_{v}=\operatorname{Ind}{ }_{B_{0}}^{M} \chi_{v}$, where $M$ is the Levi subgroup whose $L$-group is $M^{*}=\operatorname{Cent}\left(i m \phi_{\psi}^{+}, G^{*}\right)$. We note that $M$ is of the form $G L_{n_{1}} \times \cdots \times G L_{n_{k}} \times S p_{2 k}$. Keys-Shahidi pairing $[\mathbf{K e}-\mathbf{S h}]$ gives a desired multiplicity formula (3.2). We note that the method of $[\mathbf{K i}-\mathbf{S h}]$ can only prove "if" part in (3.2). We need the inner product of pseudo-Eisenstein series to prove "if and only if."

In general case, the Knapp-Stein $R$-groups are not enough. Our result is that the local component of the residual spectrum $C_{\text {res } v}$ is parametrized by the unipotent orbits $O_{i}, i=0, \ldots, k$ and [Springer $\left(O_{0}\right) \times \cdots \times \operatorname{Springer}\left(O_{k}\right)$ ], where [ ] is defined as follows (see Theorem 6.2.2): If $\mu_{1 v}=\mu_{2 v} \neq \mu_{i v}$ for $i=3, \ldots, k$, then we replace Springer $\left(O_{1}\right) \times \operatorname{Springer}\left(O_{2}\right)$ by

$$
C\left(O_{1}, O_{2}, \mu_{1 v}\right)=\left\{\eta \in \operatorname{Springer}(O):\left.\eta\right|_{A\left(O_{i}\right)} \in \operatorname{Springer}\left(O_{i}\right) \text {, for } i=1,2\right\} \text {, }
$$

where $O$ is a unipotent orbit of $\operatorname{Sp}\left(2\left(r_{1}+r_{2}\right), \mathbb{C}\right)$ by combining $O_{1}, O_{2}$. In Section 6, we show that the calculation of the residues of the Eisenstein series is reduced to that of the Eisenstein series of $O_{2 r_{1}} \times \cdots \times O_{2 r_{k}} \times S p_{2 r_{0}}$ attached to the trivial character. There remains a local problem of calculating the image of the normalized local intertwining operators. This has been taken care of by $[\mathbf{J a}-\mathbf{K i}]$, under some assumptions that Barbasch-Moy's result [B-Mo2] holds for disconnected group $O_{2 n}$. Therefore for symplectic groups, the result is not complete. We review the result in Section 6 .

In Section 3, we review quadratic unipotent Arthur parameters and Moeglin's reformulation of Arthur's conjecture on the multiplicity formula. In Section 4, we review Eisenstein series and pseudo-Eisenstein series in the sense of [M-W1] attached to a character of a Borel subgroup. In Section 7, we show how automorphic representations obtained by Kudla-Rallis $[\mathbf{K u}-\mathbf{R a}]$ by considering degenerate principal series, are fit as a special case. In Section 8, we explain how the result in $[\mathbf{K i}-\mathbf{S h}]$ is fit as a special case.

Acknowledgments. We would like to thank Prof. Shahidi. Without his guidance and encouragement this paper could not have been finished. We would like to thank Prof. Moeglin for patiently answering many of our questions [M5] and for many correspondences. We also thank Dr. Jantzen for many discussions. Without his help on local questions [Ja-Ki], this paper could not have been finished. 


\section{Preliminaries.}

Let $F$ be a field and let $G=S O_{2 n+1}, S p_{2 n}$ or $S O_{2 n}$ over $F$. Let $J_{n}$ be the $n \times n$ matrix given by

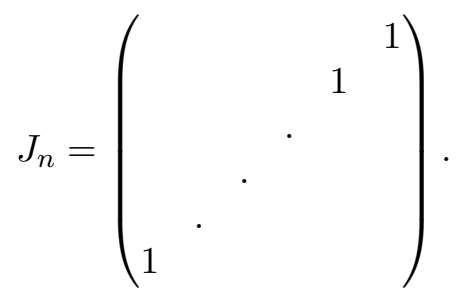

Let $J_{2 n}^{\prime}=\left(\begin{array}{cc} & J_{n} \\ -J_{n} & \end{array}\right)$. Then

$$
S p(2 n)=\left\{\left.g \in G L(2 n)\right|^{t} g J_{2 n}^{\prime} g=J_{2 n}^{\prime}\right\},
$$

and

$$
S O(n)=\left\{\left.g \in G L(n)\right|^{t} g J_{n} g=J_{n} ; \operatorname{det}(g)=1\right\} .
$$

In each case we let $T$ be the maximal split torus consisting of diagonal matrices in $G$. Then

$$
T(F)=\left\{t\left(l_{1}, \ldots, l_{n}\right)=\left(\begin{array}{ccccccc}
l_{1} & & & & & & \\
& l_{2} & & & & & \\
& & \ddots & & & & \\
& & & l_{n} & & & \\
& & & l_{n}^{-1} & & \\
& & & & \ddots & & \\
& & & & & l_{2}^{-1} & \\
& & & & & & l_{1}^{-1}
\end{array}\right) \mid l_{i} \in F^{*}\right\},
$$

if $G=S p(2 n)$ or $S O(2 n)$, and

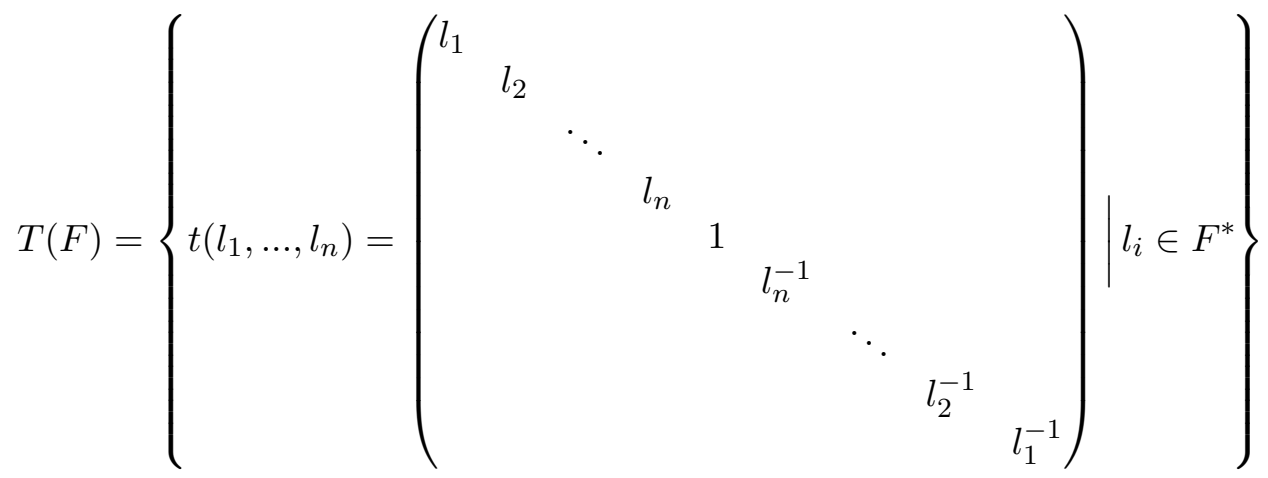

if $G=S O(2 n+1)$.

Let $\Phi(G, T)$ be the roots of $G$ with respect to $T$. We choose the ordering on the roots so that the Borel subgroup $B$ is the subgroup of upper triangular 
matrices in $G$. Let $\Delta$ be the simple roots in $\Phi(G, T)$ given by $\Delta=\left\{\alpha_{j}\right\}_{j=1}^{n}$, with $\alpha_{j}=e_{j}-e_{j+1}$ for $1 \leq j \leq n-1$, and

$$
\alpha_{n}= \begin{cases}e_{n} & G=S O(2 n+1), \\ 2 e_{n} & G=S p(2 n), \\ e_{n-1}+e_{n} & G=S O(2 n) .\end{cases}
$$

We let $\langle$,$\rangle be the standard Euclidean inner product on \Phi(G, T)$. If $\Phi$ is a root system of type $B_{n}, C_{n}$, or $D_{n}$, then we denote by $G(\Phi)$ the split group with root system $\Phi$.

For $G=S O(2 n+1)$ or $S p(2 n)$, the Weyl group $W(G / T) \simeq S_{n} \ltimes \mathbb{Z}_{2}^{n} . S_{n}$ acts by permutations on the $\lambda_{i}, i=1, \ldots, n$. We will use standard cycle notation for the elements of $S_{n}$. Thus $(i j)$ interchanges $\lambda_{i}$ and $\lambda_{j}$. If $c_{i}$ is the non-trivial element in the $i$-th copy of $\mathbb{Z}_{2}$ then $c_{i}$ takes $\lambda_{i}$ to $\lambda_{i}^{-1}$. The element $c_{i}$ is called a sign change because its action on $\Phi(G, T)$ takes $e_{i}$ to $-e_{i}$. For $G=S O(2 n)$, the Weyl group is given by $W(G / T) \simeq S_{n} \ltimes Z_{2}^{n-1}$. $S_{n}$ acts by permutations on the $\lambda_{i}$, and $\mathbb{Z}_{2}^{n-1}$ acts by even numbers of sign changes. The requirement that the number of sign changes be even comes from the determinant condition in $S O(2 n)$. Note that the sign change $c_{i}$ is an element of $O(2 n)$ and normalizes $T(F)$. Each $c_{i}$ acts on $S O(2 n)$ by conjugation, and $c_{n}$ induces the non-trivial graph automorphism on the Dynkin diagram of $\Phi(G, T)$.

\section{Unipotent orbits of classical groups over $\mathbb{C}$.}

Theory of Jordan normal forms implies that a unipotent matrix in $G L_{N}$ is conjugate to $J\left(p_{1}\right) \oplus J\left(p_{2}\right) \oplus \cdots \oplus J\left(p_{s}\right), p_{1} \geq p_{2} \geq \cdots \geq p_{s}, p_{1}+p_{2}+\cdots+p_{s}=$ $N$, where $J(p)$ is the $p \times p$ Jordan matrix with entries 1 just above the diagonal and the diagonal and zero everywhere else. Therefore unipotent classes in $G L_{N}$ are in 1 to 1 correspondence with partitions $\lambda$ of $N$. We use the following standard notation for $\lambda: \lambda=\left(1^{r_{1}}, 2^{r_{2}}, 3^{r_{3}}, \ldots\right)$, where $r_{j}$ is the number of $p_{i}$ equal to $j$.

Let $G$ be a classical group, of type $B_{n}\left(O_{2 n+1}(\mathbb{C})\right), C_{n}\left(S p_{2 n}(\mathbb{C})\right)$ or $D_{n}$ $\left(O_{2 n}(\mathbb{C})\right)$. We start with the following facts:

(1) $X, X^{\prime} \in G$ are conjugate in $G$ if and only if they are conjugate in $G L_{N}, N=2 n+1$ or $2 n$.

(2) Let $X \in G L_{N}$ be unipotent. Then $X$ is conjugate to an element of $G$ if and only if $r_{i}$ is even for even $i$ in the orthogonal case and for odd $i$ in the symplectic case.

Therefore for $G=O_{2 n+1}(\mathbb{C})$, unipotent classes are in 1 to 1 correspondence with partitions $\lambda$ of $2 n+1$ such that $r_{i}$ is even for even $i$.

Let $u$ be a unipotent element in $G$ and let $S_{u}$ be its centralizer in $G$. Then we have: 
(3) In the orthogonal case (resp. symplectic) case, $S_{u} / S_{u}^{\circ}$ is $k$ product of $\mathbb{Z} / 2 \mathbb{Z}$, where $k$ is the number of odd (resp. even) $i$ such that $r_{i}>0$.

Here we note that for $G=G L_{N}(\mathbb{C})$, the centralizer $Z_{G}(S)$ is connected for any subset $S$ of $G$.

We say that a unipotent element $u$ is distinguished if all maximal tori of Cent $(u, G)$ are contained in the center of $G^{\circ}$, the connected component of the identity. This is equivalent to the fact that the unipotent orbit $O$ of $u$ does not meet any proper Levi subgroup of $G$ (Spaltenstein [Sp, p. 67]). (I.e., if $L$ is a Levi subgroup of a parabolic subgroup of $G$ and $u \in L$ for a $u \in O$, then $L^{\circ}=G^{\circ}$.) If $G=O_{2 n+1}(\mathbb{C})$, then $G^{\circ}=S O_{2 n+1}(\mathbb{C})$ and $G^{\circ}$ has trivial center. By Carter $[\mathbf{C}]$, for $G=O_{2 n+1}(\mathbb{C})$ or $O_{2 n}(\mathbb{C})$, if $u$ is a unipotent element with Jordan blocks $\left(1^{r_{1}}, 2^{r_{2}}, \ldots\right)$, then the reductive part of the connected centralizer $\operatorname{Cent}(u, G)^{\circ}$ is of type

$$
\prod_{i \text { even }} C_{r_{i} / 2} \times \prod_{i \text { odd }, r_{i} \text { even }} D_{r_{i} / 2} \times \prod_{i \text { odd }, r_{i} \text { odd }} B_{\left(r_{i}-1\right) / 2} .
$$

Therefore, $O$ is a distinguished unipotent class if and only if it has Jordan blocks $\left(1^{r_{1}}, 3^{r_{3}}, 5^{r_{5}}, \ldots\right)$, where $r_{i}=0$ or 1 .

Jacobson-Morozov Theorem. Suppose $u$ is a unipotent element in a semi-simple algebraic group $G$. Then there exists a homomorphism $\phi$ : $S L_{2} \longmapsto G$ such that $\phi\left(\begin{array}{ll}1 & 1 \\ 0 & 1\end{array}\right)=u$.

Here, replacing $\phi$ by a conjugate under $G$, we can assume that $\phi\left(\begin{array}{cc}a & 0 \\ 0 & a^{-1}\end{array}\right)$ is in the closure of the positive Weyl chamber in the maximal torus. In fact, by the theory of weighted Dynkin diagrams (cf. Section 5.6 of $[\mathbf{C}]$ ), $\phi\left(\begin{array}{cc}a & 0 \\ 0 & a^{-1}\end{array}\right)$ is uniquely determined by the unipotent orbit of $u$ as follows (Carter [C, p. 395]):

Suppose $O$ has Jordan blocks $\left(d_{1}, d_{2}, d_{3}, \ldots\right)$. For each $d_{i}$, we take the set of integers $d_{i}-1, d_{i}-3, \ldots, 3-d_{i}, 1-d_{i}$. We then take the union of these sets for all $d_{i}$ and write this union as $\left(\xi_{1}, \xi_{2}, \xi_{3}, \ldots\right)$ with $\xi_{1} \geq \xi_{2} \geq \xi_{3} \geq \ldots$. Then

$$
\phi\left(\begin{array}{cc}
a & 0 \\
0 & a^{-1}
\end{array}\right)=\operatorname{diag}\left(a^{\xi_{1}}, a^{\xi_{2}}, a^{\xi_{3}}, \ldots\right) .
$$

Lemma ([B-V, Prop. 2.4]). Let $u$ be a unipotent element and $\phi: S L_{2} \longmapsto$ $G$ be a homomorphism such that $\phi\left(\begin{array}{ll}1 & 1 \\ 0 & 1\end{array}\right)=u$. Let $S_{\phi}=\operatorname{Cent}(i m \phi, G) \subset$ $S_{u}=\operatorname{Cent}(u, G)$ and $U^{u}$ be the unipotent radical of $S_{u}$. Then:

(1) $S_{u}=S_{\phi} \cdot U^{u}$, a semi-direct product. $S_{\phi}$ is reductive. 
(2) The inclusion $S_{\phi} \subset S_{u}$ induces an isomorphism between $S_{\phi} / S_{\phi}^{\circ} Z_{G}$ and $S_{u} / S_{u}^{\circ} Z_{G}$.

\section{Quadratic unipotent Arthur parameters.}

We follow Moeglin [M3]. Let $F$ be a number field and let $W_{F}$ be the global Weil group of $F$. Let $G=S p_{2 n}, S O_{2 n+1}, O_{2 n}$ and we can take the dual group $G^{*}=O_{2 n+1}(\mathbb{C}), S p_{2 n}(\mathbb{C}), O_{2 n}(\mathbb{C})$. An Arthur parameter is a homomorphism

$$
\psi: W_{F} \times S L_{2}(\mathbb{C}) \longmapsto G^{*},
$$

with the following properties: (The usual definition of Arthur parameter uses Langlands' hypothetical group $L_{F}$ instead of $W_{F}$. But since we are only dealing with Langlands' quotients which come from principal series, $W_{F}$ is enough.)

(1) $\psi\left(W_{F}\right)$ is bounded and included in the set of semi-simple elements of $G^{*}$.

(2) The restriction of $\psi$ to $S L_{2}(\mathbb{C})$ is algebraic.

(3) Composing $\left.\psi\right|_{W_{F}}$ with the determinant of $G^{*}$ gives a quadratic character of $W_{F}$, denoted by $\operatorname{det} \psi$. We want $\operatorname{det} \psi=1$.

We call an Arthur parameter quadratic unipotent if the following condition is satisfied:

(4) $\left.\psi\right|_{W_{F}}$ is trivial on the intersection of the kernels of the quadratic characters of $W_{F}$.

Because of conditions (1), and (4), the action of $\psi\left(W_{F}\right)$ gives an orthogonal decomposition:

$$
\mathbb{C}^{2 n+1}, \mathbb{C}^{2 n}=V_{1} \oplus \cdots \oplus V_{k} \oplus V_{0},
$$

where $\operatorname{dim} V_{0}=2 r_{0}+1$ or $2 r_{0}, \operatorname{dim} V_{i}=2 r_{i}, 2 r_{0}+1+2 r_{1}+\cdots+2 r_{k}=$ $2 n+1,2 n, r_{1} \geq \cdots \geq r_{k}$ and $V_{i}$ is the eigenspace with eigenvalue $\mu_{i}$. Here $\mu_{1}, \ldots, \mu_{k}$ are non-trivial distinct quadratic grössencharacters of $F$, viewed as characters of $W_{F}$, and $\operatorname{dim} V_{i}, i=1, \ldots, k$, being even comes from condition (3).

The parameter $\psi$ factors through $\prod_{i=0}^{k} G_{i}^{*}$, where

$$
\begin{gathered}
G_{i}^{*}=\left\{\begin{array}{ll}
O\left(V_{i}\right), & \text { if } G=S p_{2 n}, O_{2 n} \\
S p\left(V_{i}\right), & \text { if } G=S O_{2 n+1}
\end{array}:\right. \\
\psi: W_{F} \times S L_{2}(\mathbb{C}) \longmapsto \prod_{i=0}^{k} G_{i}^{*} .
\end{gathered}
$$

(1) $W_{F}$ is mapped into the product of centers of $G_{i}^{*}$ $\psi \mid W_{F}: w \longmapsto 1 \times \mu_{1}(w) \times \cdots \times \mu_{k}(w) \in\{ \pm 1\} \times\{ \pm 1\} \times \cdots \times\{ \pm 1\}$, where $\{ \pm 1\}$ is the center of $G_{i}^{*}$, for $i=0, \ldots, k$. 
(2) By Jacobson-Morozov theorem, $\left.\psi\right|_{S L_{2}(\mathbb{C})}$ defines a unipotent orbit of $G^{*}$ of the form

$$
\prod_{i=0}^{k} O_{i}
$$

where $O_{i}$ is a unipotent orbit of $G_{i}^{*}$. Inside $O_{i}$ we fix an element $u_{i}$ such that

$$
\psi\left(\begin{array}{ll}
1 & 1 \\
0 & 1
\end{array}\right)=\prod_{i=0}^{k} u_{i}
$$

Let $S_{\psi}=\operatorname{Cent}\left(i m \psi, G^{*}\right)$ and

$$
C_{\psi}=S_{\psi} / S_{\psi}^{\circ} Z_{G^{*}}
$$

We know that $S_{\psi}$ is a maximal reductive subgroup of $\prod_{i=0}^{k} \operatorname{Cent}\left(u_{i}, G_{i}^{*}\right)$. Therefore $S_{\psi}^{\circ}=1$, i.e., $S_{\psi}$ is finite if and only if each $u_{i}$ is a distinguished unipotent element in $G_{i}^{*}$. Especially, since $\mathrm{O}_{2}(\mathbb{C})$ has no distinguished unipotent element, we have:

Lemma. Let $\psi$ be a quadratic unipotent Arthur parameter. Suppose $S_{\psi}^{\circ}=1$. Then $r_{k} \geq 2$.

Now it is clear that $S_{\psi} / S_{\psi}^{\circ} Z_{G^{*}}$ is equal to

$$
\operatorname{Cent}\left(u_{0}, G_{0}^{*}\right) / \operatorname{Cent}\left(u_{0}, G_{0}^{*}\right)^{\circ} Z_{G_{0}^{*}} \prod_{i=1}^{k} \operatorname{Cent}\left(u_{i}, G_{i}^{*}\right) / \operatorname{Cent}\left(u_{i}, G_{i}^{*}\right)^{\circ} .
$$

Here Cent $\left(u_{i}, G_{i}^{*}\right) / \operatorname{Cent}\left(u_{i}, G_{i}^{*}\right)^{\circ}$ is $t$ product of $\mathbb{Z} / 2 \mathbb{Z}$, where $t$ is the number of $i$ with $r_{i}>0$ in Jordan blocks.

For each place $v$ of $F$, we have a map $\psi_{v}=\left.\psi\right|_{W_{F_{v}} \times S L_{2}(\mathbb{C})}$. As in global case, we can then define $S_{\psi_{v}}$. But in the local case, $\mu_{i v}$ may not be distinct. Suppose $\mu_{1 v}=\mu_{2 v} \neq \mu_{i v}$ for $i=3, \ldots, k$ and $\mu_{1 v} \neq 1$. Then in the above formula,

$$
\operatorname{Cent}\left(u_{1}, G_{1}^{*}\right) / \operatorname{Cent}\left(u_{1}, G_{1}^{*}\right)^{\circ} \times \operatorname{Cent}\left(u_{2}, G_{2}^{*}\right) / \operatorname{Cent}\left(u_{2}, G_{2}^{*}\right)^{\circ}
$$

must be replaced by

$$
\operatorname{Cent}\left(u_{1} \times u_{2}, G_{12}^{*}\right) / \operatorname{Cent}\left(u_{1} \times u_{2}, G_{12}^{*}\right)^{\circ},
$$

where $G_{12}^{*}=O\left(V_{1} \oplus V_{2}\right)$ or $S p\left(V_{1} \oplus V_{2}\right)$. Now we recall Moeglin's reformulation of Arthur's conjecture ([M3]): It is a part of local Arthur's conjecture that for each irreducible character $\eta_{v}$ of $C_{\psi_{v}}$, there exists an irreducible representation $\pi\left(\psi_{v}, \eta_{v}\right)$. For each $v$, let $\Pi_{\psi_{v}}$ be the set of $\pi\left(\psi_{v}, \eta_{v}\right)$.

We define the global Arthur packet $\Pi_{\psi}$ to be the set of irreducible representations $\pi=\otimes_{v} \pi_{v}$ of $G(\mathbb{A})$ such that for each $v, \pi_{v}$ belongs to $\Pi_{\psi_{v}}$. 


\section{Arthur's Conjecture (Global).}

(1) The representations in the packet corresponding to $\psi$ may occur in the discrete spectrum if and only if $S_{\psi}$ is finite, i.e., $S_{\psi}^{\circ}=1$. We call such an Arthur parameter elliptic.

(2) For an elliptic Arthur parameter $\psi$, any $\pi \in \Pi_{\psi}$ occurs in $L_{\mathrm{dis}}^{2}(G(F) \backslash$ $G(\mathbb{A}))$ if and only if

$$
\sum_{x \in C_{\psi}} \prod_{v} \eta_{v}\left(x_{v}\right) \neq 0
$$

where $\pi=\otimes_{v} \pi\left(\psi_{v}, \eta_{v}\right), x=\left(x_{v}\right)$. Note that, if $C_{\psi}$ is abelian, (3.1) is equivalent to

$$
\left.\prod_{v} \eta_{v}\right|_{C_{\psi}}=1
$$

when $\left.\psi\right|_{W_{F}}$ is trivial.

We define

$$
L^{2}(G(F) \backslash G(\mathbb{A}))_{\psi}=\Pi_{\psi} \cap L_{\text {dis }}^{2}(G(F) \backslash G(\mathbb{A})) .
$$

Remark 3.1. For split classical groups, $C_{\psi}$ is abelian and Moeglin [M2] proved the multiplicity formula (3.2). However, in general, $C_{\psi}$ is not abelian. For example, in the case of split exceptional group of type $G_{2}, C_{\psi}$ can be $S_{3}$, the symmetric group on 3 letters. This fact leads to a "bizarre" multiplicity formula in Moeglin-Waldspurger's work on the residual spectrum of $G_{2}[\mathrm{M}-\mathrm{W} 1, \mathbf{K i 2}]$.

Let $\Pi_{\text {res } v}$ be the subset of $\Pi_{\psi_{v}}$ which consists of the local components of the residual spectrum. It is of great importance to parametrize the elements in $\Pi_{\text {res } v}$ and prove the multiplicity formula (3.1), i.e., we will construct a set of characters $C_{\text {res }_{v}} \subset \widehat{C_{\psi_{v}}}$ and each character of $C_{\text {res }_{v}}$ gives an element in $\Pi_{\mathrm{res}_{v}}$.

Remark 3.2. To any Arthur parameter $\psi$, Arthur associates a Langlands' parameter $\phi_{\psi}: W_{F} \longmapsto G^{*}$ as follows:

$$
\phi_{\psi}(w)=\psi\left(w,\left(\begin{array}{cc}
|w|^{\frac{1}{2}} & 0 \\
0 & |w|^{-\frac{1}{2}}
\end{array}\right)\right) .
$$

Let $S_{\phi_{\psi}}=\operatorname{Cent}\left(i m \phi_{\psi}, G^{*}\right)$ and $C_{\phi_{\psi}}=S_{\phi_{\psi}} / S_{\phi_{\psi}}^{\circ} Z_{G^{*}}$. For each place $v$, we have $S_{\phi_{\psi_{v}}}, C_{\phi_{\psi_{v}}}$. For each $v$, there is a natural surjection $C_{\psi_{v}} \rightarrow C_{\phi_{\psi_{v}}}$. The parameter $\phi_{\psi_{v}}$ gives a $L$-packet $\Pi_{\phi_{\psi_{v}}}$ which consists of Langlands' quotients. It is a part of Arthur's original local conjecture that for each place $v$, there is a pairing $\langle$,$\rangle on C_{\phi_{\psi_{v}}} \times \Pi_{\phi_{\psi_{v}}}$ and an enlargement $\Pi_{\psi_{v}}$ of $\Pi_{\phi_{\psi_{v}}}$ which allows an extension of $\langle$,$\rangle to C_{\psi_{v}} \times \Pi_{\psi_{v}}$ such that $\pi \in \Pi_{\phi_{\psi_{v}}} \subset \Pi_{\psi_{v}}$ if and only if the function $\langle; \pi\rangle$ lies in the image of $\widehat{C_{\phi_{\psi_{v}}}}$ in $\widehat{C_{\psi_{v}}}$. Since $C_{\psi_{v}}$ is 
abelian in our case, giving a pairing between $C_{\psi_{v}}$ and $\Pi_{\psi_{v}}$ is the same as giving a character of $C_{\psi_{v}}$.

Note that in the unipotent case (Moeglin's case) where $\left.\psi\right|_{W_{F}}$ is trivial, $C_{\phi_{\psi}}=1$. Therefore, $\Pi_{\phi_{\psi_{v}}}$ consists of only one element, that is, the spherical one. So there is no non-trivial extension to $\Pi_{\psi_{v}}$.

\section{Eisenstein series attached to Borel subgroups.}

Let $\alpha^{\vee}$ be the coroot corresponding to $\alpha \in \Phi^{+}(G, T)$. Explicitly, for $\alpha=$ $e_{i}-e_{j}, \alpha^{\vee}(l)=t\left(1, \ldots, l_{i}, \ldots, l_{j}^{-1}, \ldots, 1\right) \in T(F)$ for $1 \leq i<j \leq n$. For $\alpha=e_{i}+e_{j}, \alpha^{\vee}(l)=t\left(1, \ldots, l_{i}, \ldots, l_{j}, \ldots, 1\right)$, for $1 \leq i<j \leq n$. For $\alpha=2 e_{i}$, $\alpha^{\vee}(l)=t(1, \ldots, l, \ldots, 1)$ for $1 \leq i \leq n$. Here dots represent 1.

Let $X(T)$ (resp. $X^{*}(T)$ ) be the character (resp. cocharacter) group of $T$. There is a natural pairing $\langle\rangle:, X(T) \times X^{*}(T) \longmapsto \mathbb{Z}$. For $\alpha, \beta \in \Phi(G, T)$, $\left\langle\beta, \alpha^{\vee}\right\rangle=2(\beta, \alpha) /(\alpha, \alpha)$, where $(, \quad)$ is the standard inner product in $\Phi(G, T)$. Let $\omega_{i}=e_{1}+\cdots+e_{i}$. Then $\omega_{1}, \ldots, \omega_{n}$ are the fundamental weights of $G$ with respect to $(G, T)$. (If $G=S p_{2 n}$, since $G$ is simply connected, $X(T)=\mathbb{Z} \omega_{1}+\cdots+\mathbb{Z} \omega_{n}$ and $X^{*}(T)=\mathbb{Z} \sigma_{1}^{\vee}+\cdots \mathbb{Z} \sigma_{n}^{\vee}$.) Set $\mathfrak{a}^{*}=X(T) \otimes \mathbb{R}, \mathfrak{a}_{\mathbb{C}}^{*}=X(T) \otimes \mathbb{C}$, and $\mathfrak{a}=X^{*}(T) \otimes \mathbb{R}=\operatorname{Hom}(X(T), \mathbb{R})$, $\mathfrak{a}_{\mathbb{C}}=X^{*}(T) \otimes \mathbb{C}$. The positive Weyl chamber in $\mathfrak{a}^{*}$ is

$$
\begin{aligned}
C^{+} & =\left\{\lambda \in \mathfrak{a}^{*} \mid\left\langle\lambda, \alpha^{\vee}\right\rangle>0, \quad \text { for all } \alpha \text { positive roots }\right\} \\
& =\left\{\sum_{i=1}^{n} a_{i} \omega_{i} \mid \quad a_{i}>0\right\}
\end{aligned}
$$

We fix a non-trivial additive character $\psi_{F}=\otimes_{v} \psi_{F_{v}}$ of $\mathbb{A} / F$ and let $L(z, \mu)$ be the Hecke $L$-function with the ordinary $\Gamma$-factor so that it satisfies the functional equation $L(z, \mu)=\epsilon(z, \mu) L\left(1-z, \mu^{-1}\right)$, where $\epsilon(z, \mu)=$ $\prod_{v} \epsilon\left(z, \mu_{v}, \psi_{F_{v}}\right)$ is the usual $\epsilon$-factor. If $\mu$ is the trivial character $\mu_{0}$, then we write simply $L(z)$ for $L\left(z, \mu_{0}\right)$. We have the Laurent expansion of $L(z)$ at $z=1$ :

$$
L(z)=\frac{c(F)}{z-1}+a+\cdots .
$$

4.1. Definition of Eisenstein series. For $\mu_{1}, \ldots, \mu_{n}$ grössencharacters of $F$, we define a character $\chi=\chi\left(\mu_{1}, \ldots, \mu_{n}\right)$ of $T(\mathbb{A})$ by

$$
\chi\left(\mu_{1}, \ldots, \mu_{n}\right)\left(t\left(l_{1}, \ldots, l_{n}\right)\right)=\mu_{1}\left(l_{1}\right) \cdots \mu_{n}\left(l_{n}\right) .
$$

Let $B=T U$, where $U$ is the unipotent radical. Let $I(\chi)$ be the space of functions $\Phi$ on $G(\mathbb{A})$ satisfying $\Phi(u t g)=\chi(t) \Phi(g)$ for any $u \in U(\mathbb{A})$, $t \in T(\mathbb{A})$ and $g \in G(\mathbb{A})$. Then for each $\lambda \in \mathfrak{a}_{\mathbb{C}}^{*}$, the representation of $G(\mathbb{A})$ on the space of functions of the form

$$
g \longmapsto \Phi(g) \exp \left\langle\lambda+\rho_{B}, H_{B}(g)\right\rangle, \quad \Phi \in I(\chi),
$$


is equivalent to $I(\lambda, \chi)=\operatorname{Ind}_{B \uparrow G} \chi \otimes \exp \left(\lambda, H_{B}()\right)$. We form the Eisenstein series:

$$
E(g, f, \lambda)=\sum_{\gamma \in B(F) \backslash G(F)} f(\gamma g),
$$

where $f=\Phi e^{\left\langle\lambda+\rho_{B}, H_{B}(t)\right\rangle} \in I(\lambda, \chi)$ and $\rho_{B}$ is the half-sum of positive roots, i.e., $\rho_{B}=\omega_{1}+\cdots+\omega_{n}$. It converges absolutely for $\operatorname{Re} \lambda \in C^{+}+\rho_{B}$ and extends to a meromorphic function of $\lambda$. It is an automorphic form and the constant term of $E(g, f, \lambda)$ along $B$ is given by

$$
E_{0}(g, f, \lambda)=\int_{U(F) \backslash U(\mathbb{A})} E(u g, f, \lambda) d u=\sum_{w \in W} M(w, \lambda, \chi) f(g),
$$

where $W$ is the Weyl group of $T$ and

$$
M(w, \lambda, \chi) f(g)=\int_{w U(\mathbb{A}) w^{-1} \cap U(\mathbb{A}) \backslash U(\mathbb{A})} f\left(w^{-1} u g\right) d u .
$$

Then $M(w, \lambda, \chi)$ defines an intertwining map from $I(\lambda, \chi)$ to $I(w \lambda, w \chi)$ and satisfies a functional equation of the form

$$
M\left(w_{1} w_{2}, \lambda, \chi\right)=M\left(w_{1}, w_{2} \lambda, w_{2} \chi\right) M\left(w_{2}, \lambda, \chi\right) .
$$

Let $S$ be a finite set of places of $F$, including all the archimedean places such that for every $v \notin S, \chi_{v}, \psi_{F_{v}}$ are unramified and if $f=\otimes f_{v}$, for $v \notin S$, $f_{v}$ is the unique $K_{v}$-fixed function normalized by $f_{v}\left(e_{v}\right)=1$. We have

$$
M(w, \lambda, \chi)=\otimes_{v} A\left(w, \lambda, \chi_{v}\right) .
$$

Then by applying Gindikin-Karpelevic method, we can see that for $v \notin S$,

$$
A\left(w, \lambda, \chi_{v}\right) f_{v}=\prod_{\alpha>0, w \alpha<0} \frac{L\left(\left\langle\lambda, \alpha^{\vee}\right\rangle, \chi_{v} \circ \alpha^{\vee}\right)}{L\left(\left\langle\lambda, \alpha^{\vee}\right\rangle+1, \chi_{v} \circ \alpha^{\vee}\right)} \widetilde{f}_{v},
$$

where $\widetilde{f}_{v}$ is the $K_{v}$-fixed function in the space of $I(w \lambda, w \chi)$. For any $v$, let

$$
r_{v}(w)=\prod_{\alpha>0, w \alpha<0} \frac{L\left(\left\langle\lambda, \alpha^{\vee}\right\rangle, \chi_{v} \circ \alpha^{\vee}\right)}{L\left(\left\langle\lambda, \alpha^{\vee}\right\rangle+1, \chi_{v} \circ \alpha^{\vee}\right) \epsilon\left(\left\langle\lambda, \alpha^{\vee}\right\rangle, \chi_{v} \circ \alpha^{\vee}, \psi_{F_{v}}\right)} .
$$

We normalize the intertwining operators $A\left(w, \lambda, \chi_{v}\right)$ for all $v$ by

$$
A\left(w, \lambda, \chi_{v}\right)=r_{v}(w) R\left(w, \lambda, \chi_{v}\right) .
$$

Let $R(w, \lambda, \chi)=\otimes_{v} R\left(w, \lambda, \chi_{v}\right)$ and

$$
r(w)=\Pi_{v} r_{v}(w)=\prod_{\alpha>0, w \alpha<0} \frac{L\left(\left\langle\lambda, \alpha^{\vee}\right\rangle, \chi \circ \alpha^{\vee}\right)}{L\left(\left\langle\lambda, \alpha^{\vee}\right\rangle+1, \chi \circ \alpha^{\vee}\right) \epsilon\left(\left\langle\lambda, \alpha^{\vee}\right\rangle, \chi \circ \alpha^{\vee}\right)} .
$$

Here $R(w, \lambda, \chi)$ satisfies the functional equation

$$
R\left(w_{1} w_{2}, \lambda, \chi\right)=R\left(w_{1}, w_{2} \lambda, w_{2} \chi\right) R\left(w_{2}, \lambda, \chi\right),
$$


for any $w_{1}, w_{2}$. We know, by Winarsky $[\mathbf{W}]$ for $p$-adic cases and Shahidi [Sh3, p. 110] for real and complex cases, that

$$
A\left(w, \lambda, \chi_{v}\right) \prod_{\alpha>0, w \alpha<0} L_{v}\left(\left\langle\lambda, \alpha^{\vee}\right\rangle, \chi_{v} \circ \alpha^{\vee}\right)^{-1}
$$

is holomorphic for any $v$. So for any $v, R\left(w, \lambda, \chi_{v}\right)$ is holomorphic for $\lambda$ with $\operatorname{Re}\left(\left\langle\lambda, \alpha^{\vee}\right\rangle\right)>-1$, for all positive $\alpha$ with $w \alpha<0$. For $\chi=\chi\left(\mu_{1}, \ldots, \mu_{n}\right)$,

$$
\chi \circ \alpha^{\vee}= \begin{cases}\mu_{i} \mu_{j}^{-1}, & \text { for } \alpha=e_{i}-e_{j} \\ \mu_{i} \mu_{j}, & \text { for } \alpha=e_{i}+e_{j} \text { and } i<j \\ \mu_{i}, & \text { for } \alpha=2 e_{i} \\ \mu_{i}^{2}, & \text { for } \alpha=e_{i} .\end{cases}
$$

For $\alpha \in \Phi^{+}$, let $S_{\alpha}=\left\{\lambda \in \mathfrak{a}_{\mathbb{C}}^{*} \mid\left\langle\lambda, \alpha^{\vee}\right\rangle=1\right\}$. We call $S_{\alpha}$ a singular hyperplane. We say that $E(g, f, \lambda)$ has a pole of order $n$ at $\lambda_{0}$ if $\lambda_{0}$ is the intersection of $n$ singular hyperplanes in general position on which the Eisenstein series has a simple pole.

For $\Psi \subset \Phi^{+}$, we define $r(w, \lambda, \Psi)$ by

$$
r(w, \lambda, \Psi)=\prod_{\alpha \in \Psi, w \alpha<0} \frac{L\left(\left\langle\lambda, \alpha^{\vee}\right\rangle, \chi \circ \alpha^{\vee}\right)}{L\left(\left\langle\lambda, \alpha^{\vee}\right\rangle+1, \chi \circ \alpha^{\vee}\right) \epsilon\left(\left\langle\lambda, \alpha^{\vee}\right\rangle, \chi \circ \alpha^{\vee}\right)} .
$$

Observe that we have suppressed the dependence of $r(w, \lambda, \Psi)$ on $\chi$.

4.2. Definition of pseudo-Eisenstein series. We follow Moeglin [M1] and introduce pseudo-Eisenstein series. For $T$ a maximal torus, a character $\chi$ of $T(\mathbb{A}) / T(F)$ defines a cuspidal representation of $T$. For any $w \in W$, $w T w^{-1}=T$ and so $(T, w \chi)$ is conjugate to $(T, \chi)$. Let $I(\chi)$ be the set of entire functions $\phi$ of Paley-Wiener type such that $\phi(\lambda) \in I(\lambda, \chi)$ for each $\lambda$. Let

$$
\theta_{\phi}(g)=\left(\frac{1}{2 \pi i}\right)^{n} \int_{\operatorname{Re} \lambda=\lambda_{0}} E(g, \phi(\lambda), \lambda) d \lambda,
$$

where $\lambda_{0} \in \rho_{B}+C^{+}$. Let

$$
L^{2}(G(F) \backslash G(\mathbb{A}))_{(T, \chi)},
$$

be the space spanned by $\theta_{\phi}$ for all $\phi \in I(w \chi)$ as $w \chi$ runs through all distinct conjugates of $\chi$. Let $L_{\text {dis }}^{2}(G(F) \backslash G(\mathbb{A}))_{(T, \chi)}$ be the discrete part of $L^{2}(G(F) \backslash G(\mathbb{A}))_{(T, \chi)}$. It is the set of iterated residues of $E(g, \phi(\lambda), \lambda)$ of order $n$ and the residual spectrum attached to $(T, \chi)$. In order to decompose $L_{\text {dis }}^{2}(G(F) \backslash G(\mathbb{A}))_{(T, \chi)}$, we use the inner product formula of two pseudoEisenstein series: Let $\chi$ and $\chi^{\prime}$ be conjugate characters and $\phi \in I(\chi)$, 
$\phi^{\prime} \in I\left(\chi^{\prime}\right)$. Then

$$
\begin{aligned}
\left\langle\theta_{\phi}, \theta_{\phi^{\prime}}\right\rangle & =\frac{1}{(2 \pi i)^{n}} \int_{\operatorname{Re} \lambda=\lambda_{0}} \sum_{w \in W\left(\chi, \chi^{\prime}\right)}\left(M\left(w^{-1},-w \bar{\lambda}, w \chi\right) \phi^{\prime}(-w \bar{\lambda}), \phi(\lambda)\right) d \lambda \\
& =\frac{1}{(2 \pi i)^{n}} \int_{\operatorname{Re} \lambda=\lambda_{0}} \sum_{w \in W\left(\chi, \chi^{\prime}\right)}\left(M(w, \lambda, \chi) \phi(\lambda), \phi^{\prime}(-w \bar{\lambda})\right) d \lambda
\end{aligned}
$$

where $W\left(\chi, \chi^{\prime}\right)=\left\{w \in W \mid w \chi=\chi^{\prime}\right\}$. We can assume, after conjugation, $\chi=\chi(\underbrace{\mu_{1}, \ldots, \mu_{1}}_{r_{1}}, \ldots, \underbrace{\mu_{k}, \ldots, \mu_{k}}_{r_{k}}, \underbrace{1, \ldots, 1}_{r_{0}}), r_{0}+\cdots+r_{k}=n, r_{1} \geq \cdots \geq r_{k}$, where $\mu_{i}$ 's are non-trivial distinct quadratic grössencharacters. Let $D$ be the set of distinguished coset representatives in Proposition 6.2.2. Then $\{d \chi \mid d \in D\}$ is the set of distinct conjugates of $\chi$.

Following Moeglin [M1], we consider the constant term of the pseudoEisenstein series:

$$
E_{0}^{P S}(\phi, \lambda, \chi)=\sum_{w \in W} M\left(w^{-1}, w \lambda, w \chi\right) \phi(w \lambda) .
$$

Here $\sum_{w \in W} M\left(w^{-1}, w \lambda, w \chi\right) \phi(w \lambda)$ signifies

$$
\sum_{d \in D}\left(\sum_{w \in W(\chi, d \chi)} M\left(w^{-1}, w \lambda, w \chi\right) \phi_{d}(w \lambda)\right),
$$

where $\phi_{d} \in I(d \chi)$. Here $M\left(w^{-1}, w \lambda, w \chi\right)=r\left(w^{-1}, w \lambda, w \chi\right) R\left(w^{-1}, w \lambda, w \chi\right)$. We show the following:

Lemma 4.1. (1) $r(w, \bar{\lambda}, \chi)=\overline{r(w, \lambda, \chi)}$,

(2) $r\left(w^{-1}, w \lambda, w \chi\right)=r\left(w,-\lambda, \chi^{-1}\right)$.

Proof. (1) is immediate. We note that if $\chi=\chi\left(\mu_{1}, \ldots, \mu_{n}\right)$, then $\chi^{-1}=$ $\chi\left(\mu_{1}^{-1}, \ldots, \mu_{n}^{-1}\right)$. Then

$$
r\left(w^{-1}, w \lambda, w \chi\right)=\prod_{\alpha>0, w^{-1} \alpha<0} \frac{L\left(\left\langle w \lambda, \alpha^{\vee}\right\rangle,(w \chi) \circ \alpha^{\vee}\right)}{L\left(\left\langle w \lambda, \alpha^{\vee}\right\rangle+1,(w \chi) \circ \alpha^{\vee}\right)} .
$$

Let $\gamma=-w^{-1} \alpha$. Then $\left\{\alpha>0 \mid w^{-1} \alpha<0\right\}=-\{\gamma>0 \mid w \gamma<0\}$.

If $\alpha=e_{i} \pm e_{j},\left\langle w \lambda, \alpha^{\vee}\right\rangle=(w \lambda, \alpha)=\left(\lambda, w^{-1} \alpha\right)=-(\lambda, \gamma)=-\left\langle\lambda, \gamma^{\vee}\right\rangle$. If $\alpha=2 e_{i}$, then $\left\langle w \lambda, \alpha^{\vee}\right\rangle=\frac{1}{2}(w \lambda, \alpha)=\frac{1}{2}\left(\lambda, w^{-1} \alpha\right)=-\left\langle\lambda, \gamma^{\vee}\right\rangle$. Also we can see that $w \chi \circ(w \alpha)^{\vee}=\chi \circ \alpha^{\vee}$. Therefore, $(w \chi) \circ \alpha^{\vee}=\chi \circ\left(w^{-1} \alpha\right)^{\vee}=$ $\chi \circ(-\gamma)^{\vee}=\chi^{-1} \circ \gamma^{\vee}$. Hence (2) follows.

If $\chi$ is a quadratic character, i.e., $\chi^{-1}=\chi$, then

$$
E_{0}^{P S}(\phi, \lambda, \chi)=\sum_{w \in W} r(w,-\lambda, \chi) R\left(w^{-1}, w \lambda, w \chi\right) \phi(w \lambda) .
$$


Even though Moeglin completely analyzed the continuous spectrum as well, we will only be interested in the discrete spectrum. Let $\langle,\rangle_{\text {dis }}$ be the inner product on $L_{\text {dis }}^{2}(G(F) \backslash G(\mathbb{A}))_{(T, \chi)}$. The discrete spectrum is spanned by the square integrable iterated residues of order $n$ of $E_{0}^{P S}(\phi, \lambda, \chi)$. More precisely, $E_{0}^{P S}(\phi, \lambda, \chi)$ has a simple pole on $n$ singular hyperplanes $H_{1}, \ldots, H_{n}$ which are the poles of the $L$-functions in the numerator of the normalizing factor and whose intersection is $\lambda_{0}$. Then we have to take iterated residue at $H_{1}, H_{1} \cap H_{2}, \ldots, H_{1} \cap \cdots \cap H_{n}$.

Let us summarize Moeglin's arguments. Here $\chi=1$ and we suppress it: Moeglin showed that only the points $\lambda_{\mathfrak{p}}$, where $\mathfrak{p} \in P(O)$ and $O$ is a distinguished unipotent orbit (see Section 5 ), contribute the square integrable residues. She also found $n$ singular hyperplanes $H_{1}, \ldots, H_{n}$ whose intersection is $\lambda_{\mathfrak{p}}$. In order to take the iterated residue at $H_{1}, H_{1} \cap H_{2}, \ldots, H_{1} \cap$ $\cdots \cap H_{n}$, Moeglin defined the following function, for a certain Weyl group element $w_{\mathfrak{p}}$

$$
l_{\mathfrak{p}}(\phi, \lambda)=\sum_{w \in W} r(w,-\lambda) R\left(w_{\mathfrak{p}} w^{-1}, w \lambda\right) \phi(w \lambda) .
$$

Poles of $l_{\mathfrak{p}}(\phi, \lambda)$ in a neighborhood of $\lambda_{\mathfrak{p}}$ are contained in the local intertwining operators. She showed that $l_{\mathfrak{p}}\left(\phi, \lambda_{\mathfrak{p}}\right)$ can be defined inductively by restricting $l_{\mathfrak{p}}(\phi, \lambda)$ to $H_{1}, H_{1} \cap H_{2}, \ldots, H_{1} \cap \cdots \cap H_{n} . l_{\mathfrak{p}}\left(\phi, \lambda_{\mathfrak{p}}\right)$ is the iterated residue of $E_{0}^{P S}(\phi, \lambda)$ and spans the residual spectrum $L_{\text {dis }}^{2}(G(F) \backslash G(\mathbb{A}))_{(T, 1)}$. $l_{\mathfrak{p}}\left(\phi, \lambda_{\mathfrak{p}}\right)$ belongs to $\otimes_{v}^{\prime} R_{v}\left(w_{\mathfrak{p}}, \lambda_{\mathfrak{p}}\right) I_{v}\left(\lambda_{\mathfrak{p}}\right)$. She analyzed the image of the local intertwining operator $R_{v}\left(w_{\mathfrak{p}}, \lambda_{\mathfrak{p}}\right) I_{v}\left(\lambda_{\mathfrak{p}}\right)$ and verified Arthur's multiplicity formula. We will review her result in detail in Section 5 .

In Section 6, we calculate the constant term of the pseudo-Eisenstein series for a general $\chi$ and show that we can reduce the calculation of the poles to that of the trivial character case and apply Moeglin's result.

\section{The trivial character case; summary of Moeglin's result.}

Moeglin [M1] completely analyzed the residual spectrum of $S p_{2 n}, S O_{2 n+1}$ and split $O_{2 n}$, attached to the trivial character of the maximal torus. Her results also completely describe continuous spectrum. Her results are that the residual spectrum of split classical groups attached to the trivial character of the maximal torus is parametrized by distinguished unipotent orbits $O$ in $G^{*}$ and Springer $(O)$, which is a set of characters of $A(u)$ which are in the image of the Springer correspondence and $A(u)=C(u) / C(u)^{0}$, $C(u)=\operatorname{Cent}\left(u, G^{*}\right), u \in O$. Recall that the Springer correspondence is an injective map from the set of irreducible characters of $W$, the Weyl group, into the set of pairs $(O, \eta)$, where $O$ is a unipotent orbit and $\eta$ is an irreducible character of $A(u)$. We refer [Ja-Ki] for the review of her results. We just remark that since we are only interested in discrete spectrum, we 
need to consider distinguished unipotent orbits, i.e., $r=0$. In that case we get an explicit order of the set Springer $(O)$.

Lemma 5.1. If $O=\left(q_{1}, \ldots, q_{s}\right)$ is a distinguished unipotent orbit in $O_{2 n}(\mathbb{C})$ or $O_{2 n+1}(\mathbb{C})\left(\right.$ thus $q_{i}$ odd $)$, then

$$
\mid \text { Springer }(O) \mid={ }_{s} C_{\left[\frac{s}{2}\right]} .
$$

Proof. Let $\operatorname{Symb}(O)=\left(a_{1}, \ldots, a_{s}\right)$ (see $\left[\mathrm{M} 1\right.$, p. 663]), where $a_{i}=\left[\frac{q_{i}}{2}\right]+i$. We note that $a_{i} \leq a_{i+1}-2$ for all $i$. (If we set $q_{i}=2 r_{i}+1, q_{i+1}=2 r_{i+1}+1$, $r_{i}<r_{i+1}$, then $a_{i}=r_{i}+i$ and $a_{i+1}=r_{i+1}+i+1$.)

Let $\mathcal{S}=\left(a_{1}^{\prime}, \ldots, a_{s}^{\prime}\right)$ be a class of $\operatorname{Symb}(O)$. Then it satisfies: $\left(a_{1}^{\prime}, \ldots, a_{s}^{\prime}\right)$ coincides with $\operatorname{Symb}(O)$ and $a_{i}^{\prime} \leq a_{i+2}^{\prime}-2$ for $i=1, \ldots, s-2$. For such class $\mathcal{S}$, define $\eta$, a character of $A(O)$ by: $\eta\left(\sigma\left(q_{i}\right)\right)=1$ if and only if $\left\{j: a_{j}^{\prime}=a_{i}\right\}$ contains an element which has the same parity as $i$. Then it defines a bijection between classes of $\operatorname{Symb}(O)$ and Springer $(O)$. It is clear that the number of classes of $\operatorname{Symb}(O)$ is ${ }_{s} C_{\left[\frac{s}{2}\right]}$.

If $s$ is odd, let $s=2 r+1$. Then ${ }_{s} C_{\left[\frac{s}{2}\right]}={ }_{2 r+1} C_{r} \sim 2 \frac{2^{2 r}}{\sqrt{\pi r}}$.

We interpret Moeglin's results in terms of Arthur's parameters:

Theorem 5.2 (Moeglin). Let $G=S p_{2 n}, S O_{2 n+1}, O_{2 n}$. Then the residual spectrum of $G$ attached to the trivial character of the torus is parametrized by unipotent Arthur parameters

$$
\psi: S L_{2}(\mathbb{C}) \longmapsto G^{*},
$$

which are given by distinguished unipotent orbits in $G^{*}=O_{2 n+1}(\mathbb{C}), S p_{2 n}(\mathbb{C})$, $O_{2 n}(\mathbb{C})$. More specifically, for $O$ a distinguished unipotent orbit, let $C_{\mathrm{res}}=$ Springer $(O) \subset C_{\psi_{v}}$ and $\Pi_{\mathrm{res}}=\operatorname{Unip}_{v}(O) \subset \Pi_{\psi_{v}}$ for all finite places. For each $X \in \Pi_{\text {res }}$, there is a character $\eta_{X} \in$ Springer $(O)$ and it satisfies Arthur's conjecture. i.e.,

$$
L_{\text {dis }}^{2}(G(F) \backslash G(\mathbb{A}))_{(T, 1)} \cap L^{2}(G(F) \backslash G(\mathbb{A}))_{\psi},
$$

is the set of $\pi=\otimes_{v}^{\prime} X_{v}$, where $X_{v}$ satisfies the following conditions:

(1) There exists $\mathfrak{p} \in P(O)$ such that $X_{v} \in \operatorname{Unip}_{v}(\mathfrak{p})$ for all $v$, i.e., $\eta_{X}$ factors through $\bar{A}(\mathfrak{p})$.

(2) $X_{v}$ is spherical for almost all $v$ and archimedean places.

(3) $\prod_{v} \eta_{X_{v}}$ is trivial on $A(O)$.

\section{Arbitrary character case.}

We first do the simple case to explain our method. 
6.1. The case $G=S p_{2 n}$ and $\chi=\chi(\mu, \ldots, \mu), \mu$ non-trivial and quadratic grössencharacter. Let $\Phi_{1}=\left\{e_{i} \pm e_{j}, \quad 1 \leq i<j \leq n\right\}$ and $\Phi_{2}=\left\{2 e_{i}, \quad i=1, \ldots, n\right\}$. Then for $\alpha \in \Phi_{1}, \chi \circ \alpha^{\vee}=1$ and for $\alpha \in \Phi_{2}$, $\chi \circ \alpha^{\vee}$ is non-trivial. Then the constant term of the pseudo-Eisenstein series is given by

$$
\begin{aligned}
E_{0}^{P S}(\lambda, \phi, \chi) & =\sum_{w \in W} r(w,-\lambda, \chi) R\left(w^{-1}, w \lambda, w \chi\right) \phi(w \lambda) \\
& =\sum_{w \in W} r\left(w,-\lambda, \Phi_{1}\right) r\left(w,-\lambda, \Phi_{2}\right) R\left(w^{-1}, w \lambda, w \chi\right) \phi(w \lambda) .
\end{aligned}
$$

We note that for $f \in \operatorname{Ind}_{B}^{O_{2 n}} \exp \left(\lambda, H_{B}()\right)$,

$$
E_{0}^{P S}(\lambda, f)=\sum_{w \in W} r\left(w,-\lambda, \Phi_{1}\right) R\left(w^{-1}, w \lambda\right) f(w \lambda),
$$

is a pseudo-Eisenstein series for $O_{2 n}$ attached to the trivial character of the maximal torus. Since $\chi \circ \alpha^{\vee}$ is non-trivial for $\alpha \in \Phi_{2}, r\left(w,-\lambda, \Phi_{2}\right)$ is holomorphic.

Let $O$ be a distinguished unipotent orbit in $O_{2 n}(\mathbb{C})$ and $\mathfrak{p} \in P(O)$. Let, for $\phi \in P W$,

$$
l_{\mathfrak{p}}(\lambda, \phi, \chi)=\sum_{w \in W} r(w,-\lambda, \chi) R\left(w_{\mathfrak{p}} w^{-1}, w \lambda, w \chi\right) \phi(w \lambda) .
$$

Since $r\left(w,-\lambda, \Phi_{1}\right)$ is identically zero on $V^{\prime}(\mathfrak{p})$ if $w \notin W(\uparrow, \mathfrak{p})$, the restriction of $l_{\mathfrak{p}}(\lambda, \phi, \chi)$ to $V^{\prime}(\mathfrak{p})$ is given by

$$
\left.l_{\mathfrak{p}}(\phi, \lambda, \chi)\right|_{V^{\prime}(\mathfrak{p})}=\sum_{w \in W(\uparrow, \mathfrak{p})} r\left(w,-\lambda, \Phi_{1}\right) r\left(w,-\lambda, \Phi_{2}\right) R\left(w_{\mathfrak{p}} w^{-1}, w \lambda, w \chi\right) \phi(w \lambda) .
$$

By the definition of $W(\uparrow, \mathfrak{p})$, the poles of $\left.l_{\mathfrak{p}}(\phi, \lambda, \chi)\right|_{V^{\prime}(\mathfrak{p})}$ is contained in $r\left(w,-\lambda, \Phi_{1}\right)$. Therefore we apply Moeglin's global result for $O_{2 n}$ in the trivial character case, i.e.,

$$
\left\langle\theta_{\phi^{\prime}}, \theta_{\phi}\right\rangle_{\text {dis }}=\sum_{O \subset O_{2 n}(\mathbb{C})} \sum_{\mathfrak{p} \in P(O)} c_{\mathfrak{p}}\left\langle\left\langle l_{[\mathfrak{p}]}^{\prime}\left(\phi^{\prime}, \bar{\lambda}_{\mathfrak{p}}, \chi\right), l_{[\mathfrak{p}]}\left(\phi, \lambda_{\mathfrak{p}}, \chi\right)\right\rangle\right\rangle .
$$

We have

$$
l_{\mathfrak{p}}\left(\phi, \lambda_{\mathfrak{p}}, \chi\right) \in \otimes_{v}^{\prime} R_{v}\left(w_{\mathfrak{p}}, \lambda_{\mathfrak{p}}, \chi\right) I\left(\lambda_{\mathfrak{p}}, \chi_{v}\right) .
$$

From $[\mathbf{J a}-\mathbf{K i}]$, we summarize the result on the image of the local intertwining operator $R_{v}\left(w_{\mathfrak{p}}, \lambda_{\mathfrak{p}}, \chi\right) I\left(\lambda_{\mathfrak{p}}, \chi_{v}\right)$.

Theorem 6.1.1 (Theorem 3.4.7 of [Ja-Ki] ). Let $O$ be a distinguished unipotent orbit in $O_{2 n}(\mathbb{C})$ and $\mathfrak{p} \in P(O)$. Then $R_{v}\left(w_{\mathfrak{p}}, \lambda_{\mathfrak{p}}, \chi\right) I\left(\lambda_{\mathfrak{p}}, \chi_{v}\right)$ is semisimple. Let $\operatorname{Unip}\left(\mathfrak{p}, \mu_{v}\right)$ be the set of direct summands and $\operatorname{Unip}\left(O, \mu_{v}\right)$ be the set of union of $\operatorname{Unip}\left(\mathfrak{p}, \mu_{v}\right)$. Then for each $X \in \operatorname{Unip}\left(O, \mu_{v}\right)$, there is a character $\eta_{X} \in A(O)$ such that:

(1) If $\mu_{v} \neq 1$, then $C\left(O, \mu_{v}\right)=\left\{\eta_{X} \mid X \in \operatorname{Unip}\left(O, \mu_{v}\right)\right\}=$ Springer $(O)$. 
(2) If $\mu_{v}=1$, then

$$
\begin{aligned}
C\left(O, \mu_{v}\right) & =\left\{\eta_{X} \mid X \in \operatorname{Unip}\left(O, \mu_{v}\right)\right\} \\
& = \begin{cases}\text { Springer }(O), & \text { if } O \text { does not contain } 1 \\
\text { Springer }\left(O^{\prime}\right), & \text { if } O \text { contains } 1,\end{cases}
\end{aligned}
$$

where $O^{\prime}=O-\{1\}$.

So we have:

Theorem 6.1.2. Let $\chi=\chi(\mu, \ldots, \mu), \mu$ non-trivial quadratic grössencharacter. Then the residual spectrum attached to $\chi$ is parametrized by the distinguished unipotent orbits in $O_{2 n}$. More specifically, a distinguished unipotent orbit $O \in O_{2 n}(\mathbb{C})$ and $\chi$ give a quadratic unipotent Arthur parameter $\psi$ and let $C_{\mathrm{res}_{v}}=C\left(O, \mu_{v}\right) \subset C_{\psi_{v}}$ and $\Pi_{\mathrm{res} v}=\operatorname{Unip}\left(O, \mu_{v}\right) \subset \Pi_{\psi_{v}}$ for all nonarchimedean places. For each $X \in \Pi_{\mathrm{res}_{v}}$, there is a character $\eta_{X} \in C_{\mathrm{res} v}$ and it satisfies Arthur's conjecture, i.e.,

$$
L_{\text {dis }}^{2}(G(F) \backslash G(\mathbb{A}))_{(T, \chi)} \cap L^{2}(G(F) \backslash G(\mathbb{A}))_{\psi},
$$

is the set of $\pi=\otimes_{v}^{\prime} X_{v}$, where $X_{v}$ satisfies the following conditions:

(1) There exists $\mathfrak{p} \in P(O)$ such that $X_{v} \in \operatorname{Unip}_{v}\left(\mathfrak{p}, \mu_{v}\right)$ for all $v$.

(2) $X_{v}$ is spherical for almost all $v$ and archimedean places.

(3) $\prod_{v} \eta_{X_{v}}$ is trivial on $C_{\psi}$.

6.2. General case. For $\chi$ a non-trivial character, we can assume, after conjugation, that $\chi=\chi(\underbrace{\mu_{1}, \ldots, \mu_{1}}_{r_{1}}, \ldots, \underbrace{\mu_{k}, \ldots, \mu_{k}}_{r_{k}}, \underbrace{1, \ldots, 1}_{r_{0}}), r_{0}+\cdots+r_{k}=n$, $r_{1} \geq \cdots \geq r_{k}$. We use the following notation throughout this section:

(1) If $G=S p(2 n), G^{\prime}=G_{1}^{\prime} \times \cdots \times G_{k}^{\prime} \times G_{0}^{\prime}$, where $G_{i}^{\prime}=O\left(2 r_{i}\right)$ for $i=1, \ldots, k, G_{0}^{\prime}=S p\left(2 r_{0}\right)$. Also we denote $G_{i}^{*}=O\left(2 r_{i}, \mathbb{C}\right)$ for $i=$ $1, \ldots, k, G_{0}^{*}=O\left(2 r_{0}+1, \mathbb{C}\right)$.

(2) If $G=S O(2 n+1), G^{\prime}=G_{1}^{\prime} \times \cdots \times G_{k}^{\prime} \times G_{0}^{\prime}$, where $G_{i}^{\prime}=S O\left(2 r_{i}+1\right)$ for $i=1, \ldots, k, G_{0}^{\prime}=S O\left(2 r_{0}+1\right)$. Also we denote $G_{i}^{*}=S p\left(2 r_{i}, \mathbb{C}\right)$ for $i=1, \ldots, k, G_{0}^{*}=S p\left(2 r_{0}, \mathbb{C}\right)$.

(3) If $G=O(2 n), G^{\prime}=G_{1}^{\prime} \times \cdots \times G_{k}^{\prime} \times G_{0}^{\prime}$, where $G_{i}^{\prime}=O\left(2 r_{i}\right)$ for $i=1, \ldots, k, G_{0}^{\prime}=O\left(2 r_{0}\right)$. Also we denote $G_{i}^{*}=O\left(2 r_{i}, \mathbb{C}\right)$ for $i=$ $1, \ldots, k, G_{0}^{*}=O\left(2 r_{0}, \mathbb{C}\right)$.

We recall some basic facts from $[\mathbf{K i}-\mathbf{S h}]$. Let $E(g, \phi, \lambda)$ be the Eisenstein series associated to the character $\chi$.

Proposition 6.2.1. The Eisenstein series has a pole of order $n$ only if $r_{k} \geq 2$ and $\mu_{i}$ is a quadratic grössencharacter for $i=1, \ldots, k$. 
We divide the set of positive roots $\Phi^{+}$as follows:

$$
\begin{aligned}
\Phi_{1}= & \left\{e_{i} \pm e_{j}, \quad 1 \leq i<j \leq r_{1}\right\}, \\
\Phi_{2}= & \left\{e_{r_{1}+i} \pm e_{r_{1}+j}, \quad 1 \leq i<j \leq r_{2}\right\}, \\
& \vdots \\
\Phi_{k}=\left\{e_{r_{1}+\cdots+r_{k-1}+i} \pm e_{r_{1}+\cdots+r_{k-1}+j}, \quad 1 \leq i<j \leq r_{k}\right\}, & \\
\Phi_{0}= & \left\{e_{r_{1}+\cdots+r_{k}+i} \pm e_{r_{1}+\cdots+r_{k}+j}, \quad 1 \leq i<j \leq r_{0},\right. \\
\left.2 e_{r_{1}+\cdots+r_{k}+i}, \quad i=1, \ldots, r_{0}\right\}, & \\
\Phi_{D}= & \Phi^{+}-\bigcup_{i=0}^{k} \Phi_{k} .
\end{aligned}
$$

We note that the above is for $G=S p_{2 n}$. If $G=S O_{2 n+1}$, we need to add, to $\Phi_{i}, e_{r_{1}+\cdots+r_{i-1}+j}, j=1, \ldots, r_{i}$, for $i=1, \ldots, k$ and in $\Phi_{0}$, $2 e_{r_{1}+\cdots+r_{k}+i}$ should be $e_{r_{1}+\cdots+r_{k}+i}$. If $G=O_{2 n}$, then $\Phi_{0}$ does not have the roots $2 e_{r_{1}+\cdots+r_{k}+i}, \quad i=1, \ldots, r_{0}$.

$\Phi_{1}, \ldots, \Phi_{k}$ are root systems of type $D_{n}$ and $\Phi_{0}$ is a root system of type $C_{n}$. This corresponds to the decomposition $O\left(V_{1}\right) \times \cdots \times O\left(V_{k}\right) \times O\left(V_{0}\right) \subset$ $\mathrm{O}_{2 n+1}(\mathbb{C})$.

Let $\widetilde{W}_{i}$ be the Weyl group corresponding to $\Phi_{i}$ for $i=1, \ldots, k$ and $W_{i}$ be the Weyl group of $O_{V_{i}}$ for $i=0, \ldots, k$. Then $W_{i}=\widetilde{W}_{i} c_{r_{1}+\cdots+r_{i}}$ for $i=1, \ldots, k$. Then we have $W(\chi, \chi)=W_{1} \times \cdots \times W_{k} \times W_{0}$. Let $\lambda=\lambda_{1}+$ $\cdots+\lambda_{k}+\lambda_{0}$, where $\lambda_{i}=a_{r_{1}+\cdots+r_{i-1}+1} e_{r_{1}+\cdots+r_{i-1}+1}+\cdots+a_{r_{1}+\cdots+r_{i}} e_{r_{1}+\cdots+r_{i}}$ for $i=1, \ldots, k$ and $\lambda_{0}=a_{r_{1}+\cdots+r_{k}+1} e_{r_{1}+\cdots+r_{k}+1}+\cdots+a_{n} e_{n}$.

We recall the following well-known result. (Carter $[\mathbf{C}]$.)

Proposition 6.2.2. Let $\Delta$ be a set of simple roots and $W$ be the associated $W e y l$ group. Let $w_{\alpha}$ be the simple reflection with respect to $\alpha \in \Delta$. Then $W$ is generated by the $w_{\alpha}, \alpha \in \Delta$. Let $\theta$ be a subset of $\Delta$ and $W_{\theta}$ be the subgroup of $W$ generated by the $w_{\alpha}, \alpha \in \theta$. Then each coset $w W_{\theta}$ has a unique element $d_{\theta}$ characterized by any of the following equivalent properties:

(1) $d_{\theta} \theta>0$.

(2) $d_{\theta}$ is of minimal length in $w W_{\theta}$.

(3) For any $x \in W_{\theta}, l\left(d_{\theta} x\right)=l\left(d_{\theta}\right)+l(x)$.

We apply Proposition 6.2.2 to $\Delta=\left\{e_{1}-e_{2}, \ldots, e_{n-1}-e_{n}\right\}$ and $\theta=$ $\Delta-\left\{e_{r_{1}}-e_{r_{1}+1}, e_{r_{1}+r_{2}}-e_{r_{1}+r_{2}+1}, \ldots, e_{r_{1}+\cdots+r_{k}}-e_{r_{1}+\cdots+r_{k}+1}\right\}$. Let $D$ be the set of such distinguished coset representatives. Then for $d \in D, w_{i} \in W_{i}$, $i=0, \ldots, k$, we have

$$
\begin{aligned}
& \left\{\alpha>0: d w_{1} \cdots w_{k} w_{0} \alpha<0\right\} \\
& \quad=\cup_{i=0}^{k}\left\{\alpha \in \Phi_{i}: w_{i} \alpha<0\right\} \cup\left\{\alpha \in \Phi_{D}: d w_{1} \cdots w_{k} w_{0} \alpha<0\right\} .
\end{aligned}
$$


Then the constant term of pseudo-Eisenstein series is given by

$$
\begin{aligned}
E_{0}^{P S}(\phi, \lambda, \chi)= & \sum_{w \in W} r(w,-\lambda, \chi) R\left(w^{-1}, w \lambda, w \chi\right) \phi(w \lambda) \\
= & \prod_{i=0}^{k} \sum_{w_{i} \in W_{i}} r\left(w_{i},-\lambda_{i}, \Phi_{i}\right) \\
& \cdot\left(\sum _ { d \in D } r ( d w _ { 1 } \ldots w _ { k } w _ { 0 } , - \lambda , \Phi _ { D } ) R \left(w_{0}^{-1} w_{k}^{-1} \cdots w_{1}^{-1} d^{-1}\right.\right. \\
& \left.\left.d w_{1} \cdots w_{k} w_{0} \lambda, d \chi\right) \phi\left(d w_{1} \cdots w_{k} w_{0} \lambda\right)\right) .
\end{aligned}
$$

We note that $w_{1} \cdots w_{k} \chi=\chi$. Recall that

$$
E_{0}^{P S}(\phi, \lambda, \chi)=\sum_{w \in W} r(w,-\lambda, \chi) R\left(w^{-1}, w \lambda, w \chi\right) \phi(w \lambda)
$$

actually means

$$
\sum_{d \in D}\left(\sum_{w \in W(\chi, d \chi)} r(w,-\lambda, \chi) R\left(w^{-1}, w \lambda, w \chi\right) \phi_{d}(w \lambda)\right),
$$

where $\phi_{d} \in I(d \chi)$. By the cocycle relation, we have

$$
\begin{aligned}
& R\left(w_{0}^{-1} w_{k}^{-1} \cdots w_{1}^{-1} d^{-1}, d w_{1} \cdots w_{k} w_{0} \lambda, d \chi\right) \\
& \quad=R\left(w_{0}^{-1} w_{k}^{-1} \cdots w_{1}^{-1}, w_{1} \cdots w_{k} w_{0} \lambda, \chi\right) R\left(d^{-1}, d w_{1} \cdots w_{k} w_{0} \lambda, d \chi\right) .
\end{aligned}
$$

Let

$$
\begin{aligned}
f\left(w_{1} \cdots w_{k} w_{0} \lambda\right)=\sum_{d \in D} r\left(d w_{1} \cdots w_{k} w_{0},-\lambda, \Phi_{D}\right) \\
R\left(d^{-1}, d w_{1} \cdots w_{k} w_{0} \lambda, d \chi\right) \phi\left(d w_{1} \cdots w_{k} w_{0} \lambda\right) .
\end{aligned}
$$

Then we have

$$
\begin{aligned}
E_{0}^{P S}(\phi, \lambda, \chi) & =\prod_{i=0}^{k} \sum_{w_{i} \in W_{i}} r\left(w_{i},-\lambda_{i}, \Phi_{i}\right) \\
& R\left(w_{0}^{-1} w_{k}^{-1} \cdots w_{1}^{-1} d^{-1}, d w_{1} \cdots w_{k} w_{0} \lambda, d \chi\right) f\left(w_{1} \cdots w_{k} w_{0} \lambda\right) .
\end{aligned}
$$

We note that it has the same normalizing factors as the Eisenstein series of $O_{2 r_{1}}, \ldots, O_{2 r_{k}}$ and $S p_{2 r_{0}}$ attached to the trivial character. Let $O_{i}$ 's be distinguished unipotent orbits in $O_{2 r_{i}}(\mathbb{C})$ for $i=1, \ldots, k$ and $O_{0}$ in $O_{2 r_{0}+1}(\mathbb{C})$. 
Let $\mathfrak{p}_{i} \in P\left(O_{i}\right)$ for $i=0, \ldots, k$ and $\mathfrak{p}=\mathfrak{p}_{0} \times \cdots \times \mathfrak{p}_{k}$. Let

$$
\begin{aligned}
l_{\mathfrak{p}}(\phi, \lambda, \chi)=\prod_{i=0}^{k} \sum_{w_{i} \in W_{i}} r\left(w_{i},-\lambda_{i}, \Phi_{i}\right) \\
\quad \cdot R\left(w_{\mathfrak{p}} w_{k}^{-1} \cdots w_{0}^{-1}, w_{0} \cdots w_{k} \lambda, \chi\right) f\left(w_{0} \cdots w_{k} \lambda\right) .
\end{aligned}
$$

Here $w_{\mathfrak{p}}=w_{\mathfrak{p}_{0}} \cdots w_{\mathfrak{p}_{k}}$. Since $r\left(w_{i},-\lambda_{i}, \Phi_{i}\right)$ is identically zero on $V^{\prime}\left(\mathfrak{p}_{i}\right)$ if $w_{i} \notin W\left(\uparrow, \mathfrak{p}_{i}\right)$, the restriction of $l_{\mathfrak{p}}(\phi, \lambda, \chi)$ to $V^{\prime}(\mathfrak{p})=V^{\prime}\left(\mathfrak{p}_{0}\right) \times \cdots \times V^{\prime}\left(\mathfrak{p}_{k}\right)$ is given by

$$
\begin{aligned}
\left.l_{\mathfrak{p}}(\phi, \lambda, \chi)\right|_{V^{\prime}(\mathfrak{p})}=\prod_{i=0}^{k} \sum_{w_{i} \in W\left(\uparrow, \mathfrak{p}_{i}\right)} r\left(w_{i},-\lambda_{i}, \Phi_{i}\right) \\
\quad \cdot R\left(w_{\mathfrak{p}} w_{k}^{-1} \cdots w_{0}^{-1}, w_{0} \cdots w_{k} \lambda, \chi\right) f\left(w_{0} \cdots w_{k} \lambda\right) .
\end{aligned}
$$

We note that $f\left(w_{0} \cdots w_{k} \lambda\right)$ is holomorphic on $V^{\prime}(\mathfrak{p})$. We apply Moeglin's results and define $l_{\mathfrak{p}}\left(\phi, \lambda_{\mathfrak{p}}, \chi\right)$ inductively. But the order of induction will matter. Among $V^{\prime}\left(\mathfrak{p}_{i}\right)$ 's, we can shuffle the segments. By shuffling, we mean the following:

Let $\mathfrak{p}_{1}=\left(; q_{1}, \ldots, q_{s}\right)$ and $\mathfrak{p}_{2}=\left(; q_{1}^{\prime}, \ldots, q_{t}^{\prime}\right)$ be two chains. By shuffling of $\mathfrak{p}_{1} \times \mathfrak{p}_{2}$, we mean any permutation on segments so that $(1)\left(q_{1}, q_{2}\right), \ldots$, $\left(q_{2\left[\frac{s-1}{2}\right]-1}, q_{2\left[\frac{s-1}{2}\right]}\right)$ appear in that order and $(2)\left(q_{1}^{\prime}, q_{2}^{\prime}\right), \ldots,\left(q_{2\left[\frac{t-1}{2}\right]-1}^{\prime}, q_{2\left[\frac{t-1}{2}\right]}^{\prime}\right)$ appear in that order.

Take a shuffling of segments in such a way that it satisfies a certain condition (see [Ja-Ki, (3.1)]) to produce a maximum number of components, i.e., a condition on the non-vanishing of the normalized intertwining operators.

This amounts to starting with a conjugate of $\chi$. If there is no confusion, we will still write it as $\chi$. Then

$$
\left\langle\theta_{\phi^{\prime}}, \theta_{\phi}\right\rangle_{\text {dis }}=\sum_{i=0}^{k} \sum_{O_{i}} \sum_{\mathfrak{p}} c_{\mathfrak{p}}\left\langle\left\langle l_{[\mathfrak{p}]}^{\prime}\left(\phi^{\prime}, \bar{\lambda}_{\mathfrak{p}}, \chi\right), l_{[\mathfrak{p}]}\left(\phi, \lambda_{\mathfrak{p}}, \chi\right)\right\rangle\right\rangle,
$$

where $O_{i}$ runs through distinguished unipotent orbits in $O_{2 r_{i}}(\mathbb{C})$ for $i=$ $1, \ldots, k$ and $O_{2 r_{0}+1}(\mathbb{C})$ for $i=0 . \mathfrak{p}=\mathfrak{p}_{0} \times \cdots \times \mathfrak{p}_{k} \in P\left(O_{0}\right) \times \cdots \times P\left(O_{k}\right)$. We have

$$
l_{[\mathfrak{p}]}\left(\phi, \lambda_{\mathfrak{p}}, \chi\right) \in \otimes_{v}^{\prime} R_{v}\left(w_{\mathfrak{p}}, \lambda_{\mathfrak{p}}, \chi\right) I_{v}\left(\lambda_{\mathfrak{p}}, \chi_{v}\right) .
$$

We recall the local result on the image of intertwining operators $R_{v}\left(w_{\mathfrak{p}}, \lambda_{\mathfrak{p}}\right.$, $\chi) I_{v}\left(\lambda_{\mathfrak{p}}, \chi_{v}\right)$ from $[\mathbf{J a}-\mathbf{K i}]$ : Let $\operatorname{Unip}\left(\mathfrak{p}, \chi_{v}\right)$ be the set of direct summands of $R_{v}\left(w_{\mathfrak{p}}, \lambda_{\mathfrak{p}}, \chi\right) I_{v}\left(\lambda_{\mathfrak{p}}, \chi_{v}\right)$ and $\operatorname{Unip}\left(O_{1}, \ldots, O_{k}, O_{0}, \chi_{v}\right)$ be the set of union of $\operatorname{Unip}\left(\mathfrak{p}, \chi_{v}\right)$ as $\mathfrak{p}_{i}$ runs through $P\left(O_{i}\right)$ for $i=0, \ldots, k$.

Theorem 6.2.1 (Theorem 3.4.10 of $[\mathbf{J a}-\mathbf{K i}])$. $\Pi_{\mathrm{res}_{v}}=\operatorname{Unip}\left(O_{1}, \ldots, O_{k}\right.$, $\left.O_{0}, \chi_{v}\right)$ is parametrized by $C\left(O_{1}, \ldots, O_{k}, O_{0}, \chi_{v}\right)=\left[\operatorname{Springer}\left(O_{1}\right) \times \cdots \times\right.$ Springer $\left.\left(O_{k}\right) \times \operatorname{Springer}\left(O_{0}\right)\right]$, 
where [ ] is defined as follows: If $\mu_{1 v}=\mu_{2 v} \neq \mu_{i v}$ for $i=0,3, \ldots, k$, then we replace Springer $\left(O_{1}\right) \times$ Springer $\left(O_{2}\right)$ by

$C\left(O_{1}, O_{2}, \mu_{1 v}\right)=\left\{\eta \in\right.$ Springer $(O):\left.\eta\right|_{A\left(O_{i}\right)} \in \operatorname{Springer}\left(O_{i}\right)$, for $\left.i=1,2\right\}$, where $O$ is the unipotent orbit of $G_{12}^{*}$ obtained by combining $O_{1}, O_{2}$, where

$$
G_{12}^{*}= \begin{cases}O\left(2\left(r_{1}+r_{2}\right), \mathbb{C}\right), & \text { if } G=S p(2 n), O(2 n) \\ S p\left(2\left(r_{1}+r_{2}\right), \mathbb{C}\right), & \text { if } G=S O(2 n+1) .\end{cases}
$$

Therefore we have:

Theorem 6.2.2. Let $\chi=\chi(\underbrace{\mu_{1}, \ldots, \mu_{1}}_{r_{1}}, \ldots, \underbrace{\mu_{k}, \ldots, \mu_{k}}_{r_{k}}, \underbrace{1, \ldots, 1}_{r_{0}}), r_{0}+\cdots+$ $r_{k}=n, r_{1} \geq \cdots \geq r_{k}$, where $\mu_{i}$ 's are non-trivial distinct quadratic grössencharacters. Then the residual spectrum attached to the conjugacy class of $(T, \chi)$ is parametrized by the distinguished unipotent orbits in $O_{2 r_{i}}(\mathbb{C})$, $i=1, \ldots, k$ and $O_{2 r_{0}+1}(\mathbb{C})$. More specifically, distinguished unipotent orbits $O_{i} \in O_{2 r_{i}}(\mathbb{C}), i=1, \ldots, k$ and $O_{0} \in O_{2 r_{0}+1}(\mathbb{C})$ and $\chi$ give a quadratic unipotent Arthur parameter $\psi$ and let $C_{\text {res } v}=\left[\operatorname{Springer}\left(O_{1}\right) \times \cdots \times\right.$ Springer $\left.\left(O_{k}\right) \times \operatorname{Springer}\left(O_{0}\right)\right] \subset C_{\psi_{v}}$ and $\Pi_{\mathrm{res}_{v}}=\operatorname{Unip}\left(O_{1}, \ldots, O_{k}, O_{0}, \chi_{v}\right)$ $\subset \Pi_{\psi_{v}}$ for all non-archimedean places. For each $X \in \Pi_{\mathrm{res} v}$, there is a character $\eta_{X} \in C_{r e s_{v}}$ and it satisfies Arthur's conjecture. i.e.,

$$
L_{\text {dis }}^{2}(G(F) \backslash G(\mathbb{A}))_{(T, \chi)} \cap L^{2}(G(F) \backslash G(\mathbb{A}))_{\psi},
$$

is the set of $\pi=\otimes_{v}^{\prime} X_{v}$, where $X_{v}$ satisfies the following conditions:

(1) There exists $\mathfrak{p}_{i} \in P\left(O_{i}\right), i=0, \ldots, k$ such that $X_{v} \in \operatorname{Unip}_{v}\left(\mathfrak{p}_{1}, \ldots, \mathfrak{p}_{k}\right.$, $\left.\mathfrak{p}_{0}, \chi_{v}\right)$ for all $v$.

(2) $X_{v}$ is spherical for almost all $v$ and archimedean places.

(3) $\prod_{v} \eta_{X_{v}}$ is trivial on $C_{\psi}$.

\section{Relation to Kudla-Rallis example [Ku-Ra].}

7.1. Trivial character case. Let $G=S p_{2 n}$ and $P=M N$ be the Siegel parabolic subgroup so that $M=G L_{n}$. Consider the degenerate principal series $I(s, \chi)=\operatorname{Ind}_{P}^{G} \chi \otimes \exp \left(s, H_{P}()\right)$, where $\chi$ is a trivial character of $G L_{n}$. Let $E(s, f, P)$ be the Eisenstein series attached $f \in I(s, \chi)$.

Then Kudla-Rallis [Ku-Ra] showed the following:

\section{Theorem ([Ku-Ra $])$.}

(1) $E(s, f, P)$ has a simple pole at $s=\left\{\hat{0}, \ldots, \rho_{n}-2, \rho_{n}-1, \rho_{n}\right\}$, where $\rho_{n}=\frac{n+1}{2}$ and $\hat{0}$ means that 0 is omitted in the case when $n$ is odd.

(2) The residue at $s=\rho_{n}$ is constant.

(3) The residue at $s=\rho_{n}-1$ is not square integrable. 
(4) The residue at $s_{i}=\rho_{n}-i$ is the direct sum $\oplus R_{n}(Q)$, where the direct sum ranges over all classes of quadratic forms $Q$ with $\operatorname{dim} Q=n+$ $1-2 s_{i}$ and $\chi_{Q}=1$. Here $R_{n}(Q)$ is obtained by theta correspondence, first by local construction and then by being put together. In this case the Hasse invariant $\chi_{Q}=1$ controls the obstruction to the existence of the global quadratic space.

Let $O=\left(q_{1}, q_{2}, 1\right), q_{1}>q_{2}>1, q_{1}+q_{2}=2 n$, be a distinguished unipotent orbit in $O_{2 n+1}(\mathbb{C})$. There are two inequivalent ordered partitions in $P(O)$, namely, $\mathfrak{p}_{1}=\left(; q_{1}, q_{2}, 1\right)$ and $\mathfrak{p}_{2}=\left(; q_{2}, 1, q_{1}\right)$. We show:

Proposition 7.1. The representations $\oplus R_{n}(Q)$ are exactly those corresponding to $\mathfrak{p}_{1}$, i.e., $l_{\left[\mathfrak{p}_{1}\right]}\left(\phi, \lambda_{\mathfrak{p}_{1}}\right)$ for $\phi \in P W$. (See Section 5$)$.

Proof. In this case, in Moeglin's notation in Section $5, \lambda_{\mathfrak{p}_{1}}=\left(\lambda_{1}, \ldots, \lambda_{n}\right)$, where $\lambda_{t}=\frac{q_{1}+1}{2}-t, 1 \leq t \leq \frac{q_{1}+q_{2}}{2}$. $w_{\mathfrak{p}_{1}}(t)=-t$ for $1 \leq t \leq \frac{q_{1}-q_{2}}{2}$ and $w_{\mathfrak{p}_{1}}\left(\frac{q_{1}-q_{2}}{2}+t\right)=n+1-t$ for $1 \leq t \leq q_{2}$. We know that $S_{\mathfrak{p}_{1}}=\{\alpha>$ $\left.0 \mid w_{\mathfrak{p}_{1}} \alpha<0,\left\langle\lambda_{\mathfrak{p}_{1}}, \alpha^{\vee}\right\rangle=1\right\}$ has $n$ elements. But we can see easily that $S_{\mathfrak{p}_{1}}$ contains $e_{i}-e_{i+1}$ for $i=1, \ldots, n-1$. This means that since the simple roots $e_{i}-e_{i+1}$ for $i=1, \ldots, n-1$, generate the Siegel parabolic subgroup, the iterated residue of the Eisenstein series on the singular hyperplanes $e_{i}-e_{i+1}=1, i=1, \ldots, n-1$, is exactly the Eisenstein series attached to the trivial character of the Siegel parabolic subgroup, which Kudla-Rallis considered.

The square integrable residues at $s=\left\{\hat{0}, \ldots, \rho_{n}-2\right\}$ exactly correspond to the partitions $\left(q_{1}, q_{2}\right), q_{1}+q_{2}=2 n, q_{1}>q_{2}>1\left(s_{i}=\rho_{n}-i\right.$ corresponds to $(2 n-2 i+1,2 i-1))$.

Since $l_{\left[\mathfrak{p}_{1}\right]}\left(\phi, \lambda_{\mathfrak{p}_{1}}\right) \in \otimes_{v}^{\prime} R_{v}\left(w_{\mathfrak{p}_{1}}, \lambda_{\mathfrak{p}_{1}}\right) I_{v}\left(\lambda_{\mathfrak{p}_{1}}\right)$, the local component of $R_{n}(Q)$ is the direct summand of the image of the intertwining operator $R_{v}\left(w_{\mathfrak{p}_{1}}\right.$, $\left.\lambda_{\mathfrak{p}_{1}}\right) I_{v}\left(\lambda_{\mathfrak{p}_{1}}\right)$ which is a sum of two irreducible representations. We note that $R_{v}\left(w_{\mathfrak{p}_{1}}, \lambda_{\mathfrak{p}_{1}}\right) I_{v}\left(\lambda_{\mathfrak{p}_{1}}\right)$ is the subrepresentation of $\operatorname{Ind} \lambda_{G L_{n}}^{G}|\operatorname{det}|^{-\frac{q_{1}-q_{2}}{4}}$. Jantzen [Ja3] gave their Langlands' data.

Theorem ([Ja3, Prop 3.10]). $R_{v}\left(w_{\mathfrak{p}_{1}}, \lambda_{\mathfrak{p}_{1}}\right) I_{v}\left(\lambda_{\mathfrak{p}_{1}}\right)=\pi_{1} \oplus \pi_{2}$. Here $\pi_{1}$ is a spherical representation which is the Langlands' quotient of $\operatorname{Ind}_{M}^{G} \lambda_{O} \times \pi$, where $\lambda_{O}$ is the conjugate of $\lambda_{\mathfrak{p}}$ which is in the closure of the positive Weyl chamber and $M=\operatorname{Cent}\left(\lambda_{O}, G^{*}\right)=F^{\times} \times \cdots \times F^{\times} \times G L_{2} \times \cdots \times G L_{2} \times S L_{2}$ and $\pi=\operatorname{Ind}_{B}^{S L_{2}} 1$ is an irreducible representation of $S L_{2}$. And $\pi_{2}$ is the Langlands' quotient of

$$
\text { Ind }{ }_{M}^{G}||^{\frac{q_{1}-1}{2}} \times \cdots \times||^{\frac{q_{2}+1}{2}} \times||^{\frac{q_{2}-1}{2}} \times||^{\frac{q_{2}-1}{2}} \times \cdots \times||^{2} \times||^{2} \times||^{1} \times \mathcal{T} \text {, }
$$

where $M=F^{\times} \times \cdots \times F^{\times} \times S p_{4}$ and $\mathcal{T}$ is the unique (irreducible) common component of Ind ${ }_{G L_{2}}^{S p_{4}}|\operatorname{det}|^{\frac{1}{2}}$ and Ind ${ }_{F \times \times S L_{2}}^{S p_{4}} 1 \times S t_{S L_{2}}$. Here $S t_{S L_{2}}$ is the Steinberg representation of $S L_{2}$. We note that $\mathcal{T}$ is tempered. 
Let $\operatorname{Unip}\left(\mathfrak{p}_{1}\right)=\left\{\pi_{1}, \pi_{2}\right\}$. Then Proposition 7.1 can be rephrased as follows:

Proposition 7.2. The representations $\oplus R_{n}(Q)$ are the set of $\pi=\otimes_{v}^{\prime} X_{v}$ which satisfies: (a) $X_{v} \in \operatorname{Unip}\left(\mathfrak{p}_{1}\right)$ for all $v$ (b) $X_{v}$ is spherical almost everywhere (c) $\prod_{v} \chi_{X_{v}}=1$, where $\chi_{X_{v}}$ is defined in Section 5.

But the non-degenerate Eisenstein series attached to the trivial character of the maximal torus has more residues. We need to consider $\mathfrak{p}_{2} \in P(O)$. In this case $\lambda_{\mathfrak{p}_{2}}=\left(\lambda_{1}, \ldots, \lambda_{n}\right)$, where $\lambda_{t}=\frac{q_{2}+1}{2}-t$ for $1 \leq t \leq \frac{q_{2}+1}{2}$ and $\lambda_{t+\frac{q_{2}+1}{2}}=\frac{q_{1}+1}{2}-t$ for $1 \leq t \leq \frac{q_{1}}{2}$. $w_{\mathfrak{p}_{2}}(t)=-t$ for all $t$ except $t=\frac{q_{2}+1}{2}$. Then

$$
R_{v}\left(w_{\mathfrak{p}_{2}}, \lambda_{\mathfrak{p}_{2}}\right) I_{v}\left(\lambda_{\mathfrak{p}_{2}}\right) \subset \operatorname{Ind}_{G L_{\frac{q_{2}+1}{2}}} \times S p_{q_{1}-1}|\operatorname{det}|^{-\frac{q_{2}-1}{4}} \times \operatorname{tr} .
$$

We have $R_{v}\left(w_{\mathfrak{p}_{2}}, \lambda_{\mathfrak{p}_{2}}\right) I_{v}\left(\lambda_{\mathfrak{p}_{2}}\right)=\pi_{1} \oplus \pi_{3}$.

Theorem ([Ja3]). $\pi_{3}$ is the Langlands' quotient of

$$
\text { Ind }_{F \times \cdots \times F^{\times} \times G L_{2} \times \cdots \times G L_{2}}||^{\frac{q_{1}-1}{2}} \times \cdots \times||^{\frac{q_{2}+3}{2}} \times||^{\frac{q_{2}}{2}} \sigma \times \cdots \times||^{\frac{1}{2}} \sigma,
$$

where $\sigma$ is the unique quotient of $\operatorname{Ind}_{F^{\times} \times F^{\times}}^{G L_{2}}||^{-\frac{1}{2}} \times||^{\frac{1}{2}}$ which is square integrable.

Let $\operatorname{Unip}\left(\mathfrak{p}_{2}\right)=\left\{\pi_{1}, \pi_{3}\right\}$. In this special case, we have:

Theorem 7.1. The residual automorphic representations attached to the trivial character of the maximal torus and the distinguished unipotent orbit $O=\left(q_{1}, q_{2}, 1\right)$ are the set of $\pi=\otimes_{v}^{\prime} X_{v}$ which satisfies:

(1) $X_{v} \in \operatorname{Unip}\left(\mathfrak{p}_{1}\right)$ for all $v$ or $X_{v} \in \operatorname{Unip}\left(\mathfrak{p}_{2}\right)$ for all $v$.

(2) $X_{v}$ is spherical for almost all $v$.

(3) $\prod_{v} \chi_{X_{v}}=1$.

7.2. $\chi=\chi(\mu, \ldots, \mu)$ case, $\mu$ a quadratic grössencharacter. In this case, Kudla-Rallis showed:

Theorem ([Ku-Ra] $)$. The degenerate Eisenstein series attached to the character $\mu$ of $G L_{n}$ has a pole at $s=\left\{\hat{0}, \ldots, \rho_{n}-2, \rho_{n}-1\right\}$ and the residue at $s_{i}=\rho_{n}-i$ is square integrable and given by the direct sum $\oplus R_{n}(Q)$, where the direct sum ranges over all classes of quadratic forms $Q$ with $\operatorname{dim} Q=(n+1)-2 s_{i}$ and $\chi_{Q}=\mu$. Again for this case, Hasse invariant gives obstruction to the existence of the global quadratic space.

These correspond to the distinguished unipotent orbits $O=\left(q_{1}, q_{2}\right)$ of $O_{2 n}(\mathbb{C})$, where $q_{1}>q_{2} \geq 1$. There is only one element in $P(O)$, namely, $\mathfrak{p}=\left(; q_{1}, q_{2}\right)$. The local components of $R_{n}(Q)$ are the direct summands of $R_{v}\left(w_{\mathfrak{p}}, \lambda_{\mathfrak{p}}, \chi_{v}\right) I\left(\lambda_{\mathfrak{p}}, \chi_{v}\right)$. Here $R_{v}\left(w_{\mathfrak{p}}, \lambda_{\mathfrak{p}}, \chi_{v}\right) I\left(\lambda_{\mathfrak{p}}, \chi_{v}\right)$ is a sum of two irreducible representations unless $\mu_{v}=1$ and $q_{2}=1$, in which case it is 
irreducible. If $\mu_{v} \neq 1$, then they are the Langlands' quotients of $\operatorname{Ind}_{M}^{G} \lambda_{O} \times$ $\pi_{i}$, where $\lambda_{O}$ is the conjugate of $\lambda_{\mathfrak{p}}$ which is in the closure of the positive Weyl chamber and $M=\operatorname{Cent}\left(\lambda_{O}, G^{*}\right)=F^{\times} \times \cdots \times F^{\times} \times G L_{2} \times \cdots \times G L_{2} \times S L_{2}$ and Ind ${ }_{B}^{M} \chi_{v}=\pi_{1} \oplus \pi_{2}$. If $\mu_{v}=1, q_{2} \neq 1$, then they are the ones in the case when $\chi_{v}=1$.

Remark 7.1. We can show that the residual spectrum corresponding to the unipotent orbits of the form $\mathfrak{p}=\left(; q_{1}, q_{2}, q_{3}\right), q_{3}>1$, can be obtained by considering the degenerate principal series of $G L_{l} \times S p_{2(n-l)}$, where $l=n-$ $\frac{q_{3}-1}{2}$. In this case, $\lambda_{\mathfrak{p}}=\left(\lambda_{1}, \ldots, \lambda_{n}\right)$, where $\lambda_{t}=\frac{q_{1}+1}{2}-t$, for $1 \leq t \leq \frac{q_{1}+q_{2}}{2}$ and $\lambda_{t+\frac{q_{1}+q_{2}}{2}}=\frac{q_{3}+1}{2}-t$, for $1 \leq t \leq \frac{q_{3}-1}{2}$. $w_{\mathfrak{p}}(t)=-t$ for $1 \leq t \leq \frac{q_{1}-q_{2}}{2}$ and $\frac{q_{1}+q_{2}}{2} \leq t \leq n, w_{\mathfrak{p}}\left(\frac{q_{1}-q_{2}}{2}+t\right)=\frac{q_{1}+q_{2}}{2}-t+1$ for $1 \leq t \leq q_{2}$.

We can show that $S_{\mathfrak{p}}=\left\{\alpha>0 \mid w_{\mathfrak{p}} \alpha<0,\left\langle\lambda_{\mathfrak{p}}, \alpha^{\vee}\right\rangle=1\right\}$ contains all simple roots except $e_{n-\frac{q_{3}-1}{2}}-e_{n-\frac{q_{3}+1}{2}}$. This means that the iterated residue of the Eisenstein series on the singular hyperplanes in $S_{\mathfrak{p}}$ is the residue of the degenerate Eisenstein series attached to the trivial character of the parabolic subgroup $P=M N, M=G L_{l} \times S p_{2(n-l)}$.

We note that the degenerate Eisenstein series attached to $G L_{n-1} \times S L_{2}$ parabolic subgroups has been studied by Jiang $[\mathbf{J i}]$.

\section{Relation to our previous work [Ki-Sh].}

Let $\chi=\chi(\underbrace{\mu_{1}, \ldots, \mu_{1}}_{r_{1}}, \ldots, \underbrace{\mu_{k}, \ldots, \mu_{k}}_{r_{k}}, \underbrace{1, \ldots, 1}_{r_{0}}), r_{0}+\cdots+r_{k}=n, r_{1} \geq \cdots \geq$ $r_{k} \geq 2$. In our previous work [Ki-Sh], we are concerned only with unipotent orbits of the form $O_{i}=\left(2 r_{i}-1,1\right)$ of $O_{2 r_{i}}(\mathbb{C}), i=1, \ldots, k$ and $O_{0}=\left(2 r_{0}+1\right)$ of $O_{2 r_{0}+1}(\mathbb{C})$, which correspond to the half-sum of positive roots of $O_{2 r_{i}}$ and $S p_{2 r_{0}}$, respectively. We showed:

Theorem ([Ki-Sh]). $C_{\phi_{\psi_{v}}}$ is the Knapp-Stein R-group of the unitary principal series Ind $_{B}^{M} \chi_{v}$, where $M$ is conjugate to $G L_{n_{1}} \times \cdots \times G L_{n_{r}} \times S p_{2 k}$. Here $n_{1}, \ldots, n_{r}$ are determined by $O_{i}$ 's. $\Pi_{\phi_{\psi_{v}}}$ is the set of the Langlands' quotients of $\operatorname{Ind}_{M}^{G} \pi_{i v} \times \lambda_{\mathfrak{p}}$, where $\operatorname{Ind}_{B}^{M} \chi_{v}=\oplus_{i} \pi_{i v} . \lambda_{\mathfrak{p}}$ is in the positive Weyl chamber of the split component of $M$.

Let $\mu_{i_{1}, v}, \ldots, \mu_{i_{l}, v}$ be the set of non-trivial distinct quadratic characters. Then $C_{\phi_{\psi_{v}}}$ is spanned by the order two elements $c_{r_{1}+\cdots+r_{i_{1}}}, \ldots, c_{r_{1}+\cdots+r_{i_{l}}}$. On the other hand, by Theorem 6.2.1 in Section 6, $C_{\operatorname{res}_{v}}=C\left(\mu_{i_{1}}\right) \times \cdots \times$ $C\left(\mu_{i_{l}}\right) \times C_{0}$, where $C\left(\mu_{i_{j}}\right) \simeq \mathbb{Z} / 2 \mathbb{Z}$ and $C_{0}$ is a set determined by $O_{i}$ 's. We note that $C_{\phi_{\psi_{v}}} \simeq C\left(\mu_{i_{1}, v}\right) \times \cdots \times C\left(\mu_{i_{l}, v}\right)$.

Example 8.1. Let $\chi=\chi(\mu, \mu, 1,1)$, where $\mu$ is a non-trivial quadratic grössencharacter. Let $O_{1}=(3,1)$ be a distinguished unipotent orbit of $O_{4}(\mathbb{C})$ and $O_{0}=(5)$ of $O_{5}(\mathbb{C})$. Then if $\mu_{v} \neq 1, C_{\text {res } v}=C_{\phi_{\psi_{v}}} \simeq \mathbb{Z} / 2 \mathbb{Z}$. 
But if $\mu_{v}=1, C_{\phi_{\psi_{v}}}=1$ and $C_{\text {res } v} \simeq \mathbb{Z} / 2 \mathbb{Z}$. Here $\Pi_{\text {res } v}$ is $\operatorname{Unip}\left(\mathfrak{p}_{2}\right)$ in Theorem 7.1 for $\mathfrak{p}_{2}=(; 3,1,5)$.

\section{Correction to our paper [Ki-Sh].}

p. 401: In the Abstract, the Introduction, and also in Theorem 5.1, by the technique used in that paper, we can only claim that $\Pi \in \Pi_{\phi_{\psi}}$ appears with multiplicity, not equal to $\frac{d_{\psi}}{\left|C_{\phi_{\psi}}\right|} \sum_{x \in C_{\phi_{\psi}}}\langle x, \Pi\rangle$, but greater than or equal to $\frac{d_{\psi}}{\left|C_{\phi_{\psi}}\right|} \sum_{x \in C_{\phi_{\psi}}}\langle x, \Pi\rangle$. We need to use the technique of pseudo-Eisenstein series in this paper to claim the assertion.

p. 415: In Theorem 4.5, $w_{0}$ is different from $w_{0}$ in Proposition 4.4. In fact, $w_{0}$ in Theorem 4.5 is given by $w_{0}=w_{1,0} \cdots w_{k, 0} w_{0,0}$, where $w_{i, 0}$ is the longest element in $W_{i}$ for $i=0, \ldots, k$.

p. 415: The local intertwining operator $R\left(w_{0}, \Lambda, \chi_{v}\right)$ is not holomorphic at $\Lambda_{0}$. We need to define $R\left(w_{0}, \Lambda_{0}, \chi_{v}\right)$ as the intertwining operator on Ind ${ }_{P}^{G} \Lambda_{0} \otimes \operatorname{Ind}_{B}^{M} \chi_{v}$. We assumed this implicitly there.

p. 418: In Lemma $4.8, w_{\Lambda_{1,0}}$ should be $w_{1,0}$, the longest element in $W_{1}$.

p. 418: In the proof of Lemma 4.8 and in Lemma 4.9, $w_{1}$ should be $w_{1,0}$.

p. 420: The sentence "The parameter $\Lambda_{0}$ may not be in the positive Weyl chamber of the split component of $M$," is false. The parameter $\Lambda_{0}$ is in the positive Weyl chamber of the split component of $M$.

\section{References}

[A1] J. Arthur, On some problems suggested by the trace formula, in 'Lie Group Representations, II,' Lecture Notes in Math., 1041, Springer-Verlag, (1984), 1-49, MR 85k:11025, Zbl 541.22011.

[A2] - Unipotent automorphic representations: conjectures, in 'Orbites Unipotentes det Représentations II. Groupes p-Adiques et Réels', Astérisque, 171-172 (1989), 13-71, MR 91f:22030, Zbl 728.22014.

[A3] Eisenstein series and the trace formula, in 'Proceedings of Symposia in Pure Mathematics', 33(I) (1979), 253-274, MR 81b:10020, Zbl 431.22016.

[B-Mo] D. Barbasch and A. Moy, A unitarity criterion for p-adic groups, Inv. Math., 98 (1988), 19-37, MR 90m:22038, Zbl 676.22012.

[B-Mo2] __ Reduction to real infinitesimal character in affine Hecke algebras, J. of the AMS, 6(3) (1993), 611-635, MR 93k:22015, Zbl 835.22016.

[B-V] D. Barbasch and D. Vogan, Unipotent representations of complex semisimple groups, Ann. of Math., 121 (1985), 41-110, MR 86i:22031, Zbl 582.22007.

[C] R. Carter, Finite Groups of Lie Type, Conjugacy Classes and Complex Characters, John Wiley and Sons, 1985, MR 87d:20060, Zbl 567.20023.

[Ja1] C. Jantzen, Degenerate principal series for symplectic groups and odd-orthogonal groups, Mem. of AMS, 124(590) (1996), MR 97d:22020, Zbl 866.22016. 
[Ja3] , Personal communication, 1995.

[Ja-Ki] C. Jantzen and H. Kim, Parametrization of the image of normalized intertwining operators, Pacific J. Math., 199 (2001), 367-415.

[Ji] D. Jiang, On the degree 16 L-function for $G S p(2) \times G S p(2)$, Mem. of AMS, 123(588) (1996).

[Ke-Sh] C.D. Keys and F. Shahidi, Artin L-functions and normalization of intertwining operators, Ann. Scient. Ec. Norm. Sup., 21 (1988), 67-89, MR 89k:22034, Zbl 654.10030.

[Ki1] H. Kim, The residual spectrum of $S p_{4}$, Comp. Math., 99(2) (1995), 129-151, MR 97c:11056, Zbl 877.11030.

[Ki2] - The residual spectrum of $G_{2}$, Can. J. Math., 48(6) (1996), 1245-1272, MR 97i:11055, Zbl 879.11024.

[Ki3] - The residual spectrum of $U(n, n)$; contribution from Borel subgroups, preprint.

[Ki-Sh] H. Kim and F. Shahidi, Quadratic unipotent Arthur parameters and residual spectrum of $S p_{2 n}$, Amer. J. Math., 118(2) (1996), 401-425, MR 98f:11048, Zbl 866.11036.

[Ki-Sh2] _ Symmetric cube L-functions of $G L_{2}$ are entire, Ann. of Math., 150(2) (1999), 645-662, MR 2000k:11065.

[Ku-Ra] S. Kudla and S. Rallis, A regularized Siegel-Weil formula; the first term identity, Ann. of Math., 140(1) (1994), 1-80, MR 95f:11036, Zbl 818.11024.

[L-La] J-P. Labesse and R.P. Langlands, L-indistinguishability for $S L(2)$, Can. J. Math., 31 (1979), 726-785, MR 81b:22017, Zbl 421.12014.

[La1] R.P. Langlands, On the Functional Equations Satisfied by Eisenstein Series, Lecture Notes in Math., 544, Springer-Verlag, 1976, MR 58 \#28319, Zbl 332.10018.

[La2] - On the classification of irreducible representations of real algebraic groups, in 'Representation Theory and Harmonic Analysis on Semisimple Lie Groups', (1989), 101-170, MR 91e:22017, Zbl 741.22009.

[La3] — Euler Products, Yale University Press, 1971, MR 54 \#7387, Zbl 231.20016.

[Lu] G. Lusztig, Intersection cohomology complexes on a reductive group, Inv. Math., 75 (1984), 205-272, MR 86d:20050, Zbl 547.20032.

[M1] C. Moeglin, Représentations unipotentes et formes automorphes de carré intégrable, Forum Mathematicum, 6 (1994), 651-744, MR 95k:22024, Zbl 816.11034.

[M2] - Orbites unipotentes et spectre discret non ramifie, Le cas des groupes classiques déployś, Comp. Math., 77 (1991), 1-54, MR 92d:11054, Zbl 809.11030.

[M3] - Une conjecture sur le spectre résiduel des groupes classiques, preprint, 1994.

[M4]_, Représentations quadratiques unipotentes des groupes classiques padiques Duke Math. J., 84 (1996), 267-332, MR 97h:22013, Zbl 864.22008.

[M5] Cuspidal quadratic unipotent representation, preprint, 1994.

[M6]_, Letters. 
[M-W1] C. Moeglin and J.L. Waldspurger, Spectral Decomposition and Eisenstein Series, une Paraphrase de l'Ecriture, Cambridge Tracts in Mathematics, 113, Cambridge University Press, 1995, MR 97d:11083, Zbl 846.11032.

[M-W2] _ Le spectre résiduel de $G L(n)$, Ann. Scient. Éc. Norm. Sup., 22 (1989), 605-674, MR 91b:22028, Zbl 696.10023.

[Sh1] F. Shahidi, Twisted endoscopy and reducibility of induced representations for padic groups, Duke Math. J., 66(1) (1992), 1-41, MR 93b:22034, Zbl 785.22022.

[Sh2] _ On the Ramanujan conjecture and finiteness of poles for certain Lfunctions, Ann. of Math., 127 (1988), 547-584, MR 89h:11021, Zbl 654.10029.

[Sh3] _ Whittaker models for real groups Duke Math. J., 47(1) (1980), 99-125, MR 81d:22020, Zbl 433.22007.

[Sp] N. Spaltenstein, Classes Unipotentes et Sous-groupes de Borel, Lecture Notes in Mathematics, 946, Springer-Verlag, 1982, MR 84a:14024, Zbl 486.20025.

[W] N. Winarsky, Reducibility of principal series representations of p-adic Chevalley groups, Amer. J. Math., 100(5) (1978), 941-956, MR 80f:22018, Zbl 475.43005.

Received June 15, 1999. This author was partially supported by NSF grant DMS-9610387.

DePT OF MATh.

SOUTHERN ILLINOIS UNIVERSITY

Carbondale, IL 62901

E-mail address: henrykim@math.siu.edu 